\title{
Sign characteristics of regular Hermitian matrix pencils under generic rank-1 and rank-2 perturbations
}

\author{
Leonhard Batzke*
}

November 20, 2014

\begin{abstract}
The spectral behavior of regular Hermitian matrix pencils is examined under certain structure-preserving rank-1 and rank-2 perturbations. Since Hermitian pencils have signs attached to real (and infinite) blocks in canonical form, it is not only the Jordan structure but also this so-called sign characteristic that needs to be examined under perturbation. The observed effects are as follows: Under a rank-1 or rank-2 perturbation, generically the largest one or two, respectively, Jordan blocks at each eigenvalue $\lambda$ are destroyed, and if $\lambda$ is an eigenvalue of the perturbation, also one new block of size one is created at $\lambda$. If $\lambda$ is real (or infinite), additionally all signs at $\lambda$ but one or two, respectively, that correspond to the destroyed blocks, are preserved under perturbation. Also, if the potential new block of size one is real, its sign is in most cases prescribed to be the sign that is attached to the eigenvalue $\lambda$ in the perturbation.
\end{abstract}

Key words. Matrix pencil, Hermitian matrix pencil, sign characteristic, rank one perturbation, rank two perturbation, generic perturbation.

AMS subject classification. 15A18, 15A21, 15A22, 15B57, 47A55.

\section{Introduction}

It is well-established that when a matrix is subjected to a generic rank-1 perturbation, its largest Jordan block at each eigenvalue is destroyed [10, 19, 20, 21, 22]. However, different results were obtained for matrices that are structured with respect to some indefinite inner product restricting the perturbations to structure-preserving ones in $[5,14,15,16$, 17] for various classes of structured matrices. In particular, since $H$-selfadjoint matrices have additional algebraic invariants to the sizes of their Jordan blocks called the sign characteristic, this sign characteristic was studied under structured perturbations in [15].

\footnotetext{
*Institut für Mathematik, MA 4-5, Technische Universität Berlin, 10623 Berlin, Germany, Email: batzke@math.tu-berlin.de.
} 
In this work, we will consider regular Hermitian matrix pencils under low-rank perturbations, since it is well-known that they also have signs attached to their Jordan blocks. In fact, any $H$-selfadjoint matrix $A$ gives rise to the equivalent Hermitian matrix pencil $\lambda H-H A$, but the converse is in general false, since given an Hermitian matrix pencil $\lambda E-A$, the matrix $E$ may well be singular.

In particular, we will tackle the following open problem: It was shown in [4] that when regular matrix pencils are subjected to low-rank perturbations, generically not only the largest Jordan blocks at each eigenvalue will be destroyed under perturbation, but also new blocks of size one may be created at certain eigenvalues. Now, when the perturbed pencil and the perturbation are both Hermitian, then in the case of real eigenvalues the newly created blocks will have signs attached to them, but it is not known by what factors these signs can be determined. This question (among others) will be answered in Section 3 in the case of rank-1 perturbations.

Analyzing the sign characteristic under perturbations is especially relevant as it relates to the properties of control systems. Since enforcing the desired property of passivity on a control system can be achieved by moving eigenvalues of a certain Hamiltonian matrix off the imaginary axis, this question has been investigated in $[18,1]$ using Hamiltonian perturbations with small norm. Interestingly, the norm of the required perturbation is strongly linked to the sign characteristic of the corresponding eigenvalues, see also [8,9].

From the canonical form of Hermitian matrix pencils [12], we extract that a Hermitian matrix pencil of rank 1 is bound to have the form $(\lambda \beta-\alpha) u u^{*}$, where $\beta$ and $\alpha$ are real parameters. Also, from the same canonical form, we read off that a Hermitian matrix pencil of rank 2 (that cannot be decomposed into the sum of Hermitian rank-1 pencils) either has the form

$$
\left[\begin{array}{lll}
u & v & w
\end{array}\right]\left[\begin{array}{lll}
0 & 0 & \lambda \\
0 & 0 & 1 \\
\lambda & 1 & 0
\end{array}\right]\left[\begin{array}{c}
u^{*} \\
v^{*} \\
w^{*}
\end{array}\right]
$$

i.e., two paired singular blocks of minimal index one, or it has the form

$$
\left[\begin{array}{ll}
u & v
\end{array}\right]\left[\begin{array}{cc}
0 & \lambda-\mu \\
\lambda-\bar{\mu} & 0
\end{array}\right]\left[\begin{array}{l}
u^{*} \\
v^{*}
\end{array}\right]
$$

i.e., a pair of nonreal eigenvalues $\mu$ and $\bar{\mu}$. In our perturbation analysis, we will consider rank-1 perturbations of the form $(\lambda \beta-\alpha) u u^{*}$ and rank-2 perturbations of the form (1.2), since the class of rank-2 perturbations (1.1) with nontrivial singular part will be more difficult to handle. A different approach that leads to these types of Hermitian perturbations will be given in Section 2.3.

The next section will cover preliminaries as versions of the partial Brunovsky form and results on the canonical form and the sign characteristic of Hermitian matrix pencils. In Section 3, we will determine the generic Jordan structure and sign characteristic of Hermitian regular matrix pencils under the above type of Hermitian rank-1 perturbations. Then, in Section 4 we repeat these steps for Hermitian rank-2 perturbations and in the final section, a brief conclusion will be presented. 
Throughout this paper, we will identify matrix pencils $\lambda E-A$ with matrix pairs $(E, A)$ using whichever notion is more convenient. Further, for square matrices $X$ and $Y$ (not necessarily of the same dimension), define $X \oplus Y:=\operatorname{diag}(X, Y)$ and let $X^{\oplus p}:=X \oplus \cdots \oplus X$ ( $p$ times). Also, denote by $e_{j, n}$ the $j$ th standard basis vector in $\mathbb{C}^{n}$, where the second index will be omitted whenever clear from the context. Finally, let us denote by $J_{n}(\lambda)$ the $n \times n$ Jordan block corresponding to the eigenvalue $\lambda$ and denote by $R_{n}$ the $n \times n$ reverse identity matrix (i.e., the matrix with ones on the leftbottom-topright diagonal and zeros elsewhere).

\section{Preliminaries}

In this paper, the following notion of genericity will be employed, letting $\mathbb{F}=\mathbb{R}$ or $\mathbb{F}=\mathbb{C}$.

Definition 2.1 1 1) $A$ set $\mathcal{A} \subseteq \mathbb{F}^{n}$ is called algebraic if there exist $p_{j}: \mathbb{F}^{n} \rightarrow \mathbb{F}, j=$ $1, \ldots, k$, depending polynomially on (the real and imaginary parts of) its arguments such that $a \in \mathcal{A}$ if and only if

$$
p_{j}(a)=0 \quad \text { for } \quad j=1, \ldots, k .
$$

2) An algebraic set $\mathcal{A} \subseteq \mathbb{F}^{n}$ is called proper if $\mathcal{A} \neq \mathbb{F}^{n}$.

3) A set $\Omega \subseteq \mathbb{F}^{n}$ is called generic if $\mathbb{F}^{n} \backslash \Omega$ is contained in a proper algebraic set.

Then, the intersection of finitely many generic sets is again generic and for an invertible matrix $X \in \mathbb{F}^{n, n}$ the set $X \Omega$ is generic if $\Omega \subseteq \mathbb{F}^{n}$ is generic. Subsets of $\mathbb{F}^{n, m}$ or $\mathbb{F}^{n, m} \times \mathbb{F}^{n, m}$ are called generic if they can be canonically identified with generic subsets of $\mathbb{F}^{n m}$ or $\mathbb{F}^{2 n m}$, respectively.

\subsection{Low-rank perturbations and partial Brunovsky forms}

In this section, we will recap condensed forms for matrix pencils under rank-1 and rank-2 perturbations. But first, let us review the canonical form of regular matrix pencils, i.e., matrix pencils $(E, A)$ with $\operatorname{det}(\lambda E-A) \not \equiv 0$, under equivalence: the Weierstra $\beta$ canonical form [6, Chapter 12].

Theorem 2.2 (Weierstraß canonical form) Let $(E, A) \in \mathbb{C}^{n, n} \times \mathbb{C}^{n, n}$ be a regular matrix pencil. Then, there exist invertible matrices $V, W \in \mathbb{C}^{n, n}$ and an $r \in \mathbb{N}$ so that

$$
V(E, A) W=\left(I_{r} \oplus N, J \oplus I_{n-r}\right)
$$

where $J \in \mathbb{C}^{r, r}$ and $N \in \mathbb{C}^{(n-r),(n-r)}$ are in Jordan canonical form and $N$ is nilpotent.

Jordan chains of regular matrix pencils are defined as follows [8]. 
Definition 2.3 Let $(E, A) \in \mathbb{C}^{n, n} \times \mathbb{C}^{n, n}$ be a regular matrix pencil. The ordered set of vectors $\left\{x_{1}, \ldots, x_{p}\right\} \subseteq \mathbb{C}^{n}$ is called a Jordan chain of length $p$ corresponding to an eigenvalue $\widehat{\lambda} \in \mathbb{C}$ of $(E, A)$ if $x_{1} \neq 0$ and:

$$
(\widehat{\lambda} E-A) x_{1}=0 \quad \text { and } \quad(\widehat{\lambda} E-A) x_{j}=-E x_{j-1}, \quad j=2, \ldots, p .
$$

Similarly, $\left\{x_{1}, \ldots, x_{p}\right\}$ is called a Jordan chain of length $p$ corresponding to $\infty$ if $x_{1} \neq 0$ and:

$$
E x_{1}=0 \quad \text { and } \quad E x_{j}=A x_{j-1}, \quad j=2, \ldots, p .
$$

Then, the following theorem is crucial for characterizing regular matrix pencils under rank-1 perturbations.

Theorem 2.4 (partial Brunovsky form [3, Theorem 2.7]) Let $(E, A) \in \mathbb{C}^{n, n} \times \mathbb{C}^{n, n}$ be regular and $\widehat{\lambda} \in \mathbb{C}$ an eigenvalue of $(E, A)$ with

$$
\begin{aligned}
& E=I_{n_{1}} \oplus \cdots \oplus I_{n_{m}} \oplus \widetilde{E} \in \mathbb{C}^{n, n}, \\
& A=J_{n_{1}}(\widehat{\lambda}) \oplus \cdots \oplus J_{n_{m}}(\widehat{\lambda}) \oplus \widetilde{A} \in \mathbb{C}^{n, n},
\end{aligned}
$$

where $n_{1} \geq \cdots \geq n_{m}>0$ such that $\widehat{\lambda}$ is not an eigenvalue of $(\widetilde{E}, \widetilde{A})$. Further, set $a:=n_{1}+\cdots+n_{m}$ and let $\alpha, \beta \in \mathbb{C}$ and $u, v \in \mathbb{C}^{n}$ with

$$
v^{T}=\left[\left(v^{(1)}\right)^{T}, \ldots,\left(v^{(m)}\right)^{T}, \widetilde{v}^{T}\right], \quad\left(v^{(j)}\right)^{T}=\left[v_{1}^{(j)}, \ldots, v_{n_{j}}^{(j)}\right] \in \mathbb{C}^{1, n_{j}}, \quad j=1, \ldots, m .
$$

If we define

$$
k_{j}:=\max \left\{k \mid v_{1}^{(j)}=v_{2}^{(j)}=\cdots=v_{k}^{(j)}=0\right\}, \quad j=1, \ldots, m,
$$

then the following statements hold:

1) There is an invertible matrix $S \in \mathbb{C}^{n, n}$ such that

$$
S\left(E+\beta u v^{T}, A+\alpha u v^{T}\right) S^{-1}=\left(E+\beta w e^{T}, A+\alpha w e^{T}\right)
$$

holds, where $w=S u$ and

$$
e^{T}=\left[\begin{array}{llll}
e_{k_{1}+1, n_{1}}^{T}, & \ldots, & e_{k_{m}+1, n_{m}}^{T}, & \widetilde{e}^{T}
\end{array}\right]
$$

for a suitable $\widetilde{e} \in \mathbb{C}^{n-a}$ defining $e_{k_{j}+1, n_{j}}=0$ if $k_{j}=n_{j}$. A matrix pencil (2.3) with $E$ and $A$ as in (2.2) and $e^{T}$ as above is said to be in partial Brunovsky form.

2) If (2.3) is regular, then it has at least $m-1$ linearly independent Jordan chains of lengths at least $n_{2}, \ldots, n_{m}$ corresponding to $\widehat{\lambda}$. If the (generic) condition that the first component of $v^{(j)}$ is nonzero for $j=1, \ldots, m$ holds, then they are given by:

$$
\begin{array}{cccc}
e_{1}-e_{n_{1}+1}, & e_{2}-e_{n_{1}+2}, & \ldots, & e_{n_{2}}-e_{n_{1}+n_{2}} ; \\
e_{1}-e_{n_{1}+n_{2}+1}, & e_{2}-e_{n_{1}+n_{2}+2}, & \ldots, & e_{n_{3}}-e_{n_{1}+n_{2}+n_{3}} ; \\
\vdots & \vdots & \ddots & \vdots \\
e_{1}-e_{n_{1}+\cdots+n_{m-1}+1}, & e_{2}-e_{n_{1}+\cdots+n_{m-1}+2}, & \ldots, & e_{n_{m}}-e_{n_{1}+\cdots+n_{m}} .
\end{array}
$$


Remark 2.5 If the generic condition from 2) is satisfied (i.e., if $v_{1}^{(1)}, \ldots, v_{1}^{(m)} \neq 0$ ), then the matrix $S$ from 1) is given by

$$
S:=\operatorname{Toep}\left(v^{(1)}\right) \oplus \cdots \oplus \operatorname{Toep}\left(v^{(m)}\right) \oplus I_{n-a},
$$

where Toep $\left(v^{(j)}\right)$ is the upper triangular $n_{j} \times n_{j}$ Toeplitz matrix with the first row $\left(v^{(j)}\right)^{T}$.

The following theorem is a generalization of Theorem 2.4 to rank- 2 perturbations. We point out that the notation $(\beta, \alpha)$ stands for the $1 \times 1$ matrix pencil $\lambda \beta-\alpha$.

Theorem 2.6 (rank-2 partial Brunovsky form) Let $(E, A) \in \mathbb{C}^{n, n} \times \mathbb{C}^{n, n}$ be regular and $\widehat{\lambda} \in \mathbb{C}$ an eigenvalue of $(E, A)$ with

$$
\begin{aligned}
E & =I_{n_{1}} \oplus \cdots \oplus I_{n_{m}} \oplus \widetilde{E} \in \mathbb{C}^{n, n}, \\
A & =J_{n_{1}}(\widehat{\lambda}) \oplus \cdots \oplus J_{n_{m}}(\widehat{\lambda}) \oplus \widetilde{A} \in \mathbb{C}^{n, n},
\end{aligned}
$$

such that $n_{1} \geq \cdots \geq n_{m}>0$ and $\widehat{\lambda}$ is not an eigenvalue of $(\widetilde{E}, \widetilde{A})$. Then, there is a generic set $\Omega \subseteq \mathbb{C}^{n} \times \mathbb{C}^{n}$ so that for all $\left(v_{1}, v_{2}\right) \in \Omega$ and $\alpha_{j}, \beta_{j} \in \mathbb{C} ; u_{j} \in \mathbb{C}^{n}$ and the rank-2 perturbation $(\Delta E, \Delta A)=\left(\beta_{1}, \alpha_{1}\right) u_{1} v_{1}^{T}+\left(\beta_{2}, \alpha_{2}\right) u_{2} v_{2}^{T}$, the following statements hold:

1) There exists an invertible matrix $S \in \mathbb{C}^{n, n}$ such that

$$
S(E+\Delta E, A+\Delta A) S^{-1}=\left(E+\left[\begin{array}{ll}
\beta_{1} w_{1} & \beta_{2} w_{2}
\end{array}\right] L, A+\left[\begin{array}{ll}
\alpha_{1} w_{1} & \alpha_{2} w_{2}
\end{array}\right] L\right),
$$

where $w_{j}=S u_{j}$ for $j=1,2$. Hereby, letting $a:=n_{1}+\cdots+n_{m}$ it is

$$
L=\left[\begin{array}{cccccccc}
e_{1, n_{1}}^{T} & 0 & e_{1, n_{3}}^{T} & 0 & e_{1, n_{5}}^{T} & \ldots & \sigma e_{1, n_{m}}^{T} & M \\
x^{T} & e_{1, n_{2}}^{T} & 0 & e_{1, n_{4}}^{T} & 0 & & \rho e_{1, n_{m}}^{T} &
\end{array}\right],
$$

for some $x=\left[x_{1}, \ldots, x_{n_{1}}\right]^{T} \in \mathbb{C}^{n_{1}} ; M \in \mathbb{C}^{2,(n-a)}$ where $(\sigma, \rho)=(1,0)$ if $m$ is odd and $(\sigma, \rho)=(0,1)$ otherwise. A matrix pencil of the form $(2.5)$ with $L$ as above is said to be in rank-2 partial Brunovsky form. We highlight that the first $n_{1}+\cdots+n_{m}$ columns of the transformed perturbation $S(\Delta E, \Delta A) S^{-1}$ are given by

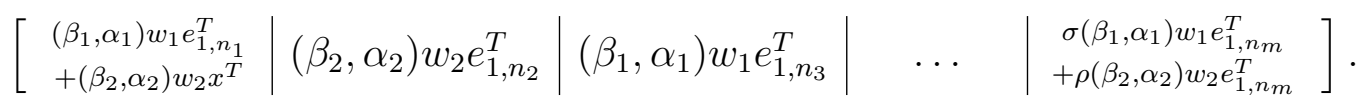

2) If $S(E+\Delta E, A+\Delta A) S^{-1}$ is regular, it has at least $m-2$ linearly independent Jordan chains corresponding to $\hat{\lambda}$ that have at least the lengths $n_{3}, n_{4}, \ldots, n_{m}$. The chain of length $n_{3}$ is given by

$$
e_{j}-e_{n_{1}+n_{2}+j}-\left[\begin{array}{lll}
e_{n_{1}+j} & \ldots & e_{n_{1}+1}
\end{array}\right]\left[\begin{array}{lll}
x_{1} & \ldots & x_{j}
\end{array}\right]^{T}, \quad j=1, \ldots, n_{3},
$$

whereas for $k=4,6, \ldots$ there are chains of length $n_{k}$ given by

$$
e_{n_{1}+j}-e_{n_{1}+\cdots+n_{k-1}+j}, \quad j=1, \ldots, n_{k},
$$

and for $k=5,7, \ldots$ there are chains of lengths $n_{k}$ given by

$$
e_{n_{1}+n_{2}+j}-e_{n_{1}+\cdots+n_{k-1}+j}, \quad j=1, \ldots, n_{k} .
$$


Proof. We target for a transformation matrix $S$ of the form

$$
S:=\left[\begin{array}{ccccc}
T_{1} & {\left[\begin{array}{c}
\widetilde{T}_{2} \\
0
\end{array}\right]} & & & \\
& T_{2} & {\left[\begin{array}{c}
\widetilde{T}_{3} \\
0
\end{array}\right]} & & \\
& T_{3} & \ddots & \\
& & \ddots & {\left[\widetilde{T}_{m}\right.} \\
0
\end{array}\right] \oplus I_{n-a}
$$

where $T_{1} \in \mathbb{C}^{n_{1}, n_{1}}$ and $\widetilde{T}_{j}, T_{j} \in \mathbb{C}^{n_{j}, n_{j}}$ for $j=2, \ldots, m$ are suitable upper triangular Toeplitz matrices. Then, by [7, Chapter 8], a matrix $S$ of this form commutes with both $E$ and $A$ since their leading $a \times a$ diagonal blocks are in Jordan form and their partitioning is conformal with that of $S$. It remains to show that under a generic condition on $\left(v_{1}, v_{2}\right)$, this $S$ can be chosen to be invertible and such that $\left[v_{1}, v_{2}\right]^{T} S^{-1}=L$ holds for some $x \in \mathbb{C}^{n_{1}}$. Partitioning $v_{j}$ as in

$$
v_{j}^{T}=\left[\begin{array}{llll}
\left(v_{j}^{(1)}\right)^{T} & \ldots & \left(v_{j}^{(m)}\right)^{T} & \widetilde{v}_{j}^{T}
\end{array}\right], \quad v_{j}^{(i)} \in \mathbb{C}^{n_{i}}, \quad i=1, \ldots, m,
$$

for $j=1,2$ and denoting by $\left(v_{j}^{(i)}\right)_{\ell}$ the $\ell$ th component of $v_{j}^{(i)}$, then $\left[v_{1}, v_{2}\right]^{T} S^{-1}=L$ is equivalent to

$$
\left[\begin{array}{cccc}
e_{1, n_{1}}^{T} T_{1} & e_{1, n_{1}}^{T}\left[\begin{array}{c}
\widetilde{T}_{2} \\
0
\end{array}\right] & e_{1, n_{3}}^{T} T_{3} \\
x^{T} T_{1} & x^{T}\left[\begin{array}{c}
\widetilde{T}_{2} \\
0
\end{array}\right]+e_{1, n_{2}}^{T} T_{2} & e_{1, n_{2}}^{T}\left[\begin{array}{c}
\widetilde{3}_{3} \\
0
\end{array}\right]
\end{array} \quad \cdots\right]=\left[\begin{array}{cccc}
\left(v_{1}^{(1)}\right)^{T} & \left(v_{1}^{(2)}\right)^{T} & \left(v_{1}^{(3)}\right)^{T} & \\
\left(v_{2}^{(1)}\right)^{T} & \left(v_{2}^{(2)}\right)^{T} & \left(v_{2}^{(3)}\right)^{T} & \cdots
\end{array}\right] .
$$

To satisfy this equation, consider that for an upper triangular Toeplitz matrix $T$, the condition $e_{1}^{T} T=v^{T}$ immediately implies $T=\operatorname{Toep}(v)$. Therefore, from the $(1,1)$ and the $(1,2)$ block of $(2.12)$ we obtain $T_{1}=\operatorname{Toep}\left(v_{1}^{(1)}\right)$ and $\widetilde{T}_{2}=\operatorname{Toep}\left(v_{1}^{(2)}\right)$, respectively. Then, assuming the generic condition $\left(v_{1}^{(1)}\right)_{1} \neq 0$ for invertibility of $T_{1}$, from the $(2,1)$ block of (2.12) we infer $x^{T}=\left(v_{2}^{(1)}\right)^{T} T_{1}^{-1}$, where the first entry of $x$ is given by $x_{1}=\left(v_{2}^{(1)}\right)_{1} /\left(v_{1}^{(1)}\right)_{1}$. Now, the equation in the $(2,2)$ block reduces to $T_{2}=$ Toep $\left(v_{2}^{(2)}-\left[\widetilde{T}_{2}^{T}, 0\right] x\right)$ and all other block equations are easily soluble as well.

Finally, $S$ is invertible if and only if the diagonal entries of $T_{1}, \ldots, T_{m}$ are nonzero. Letting $\left(v_{1}^{(1)}\right)_{1} \neq 0$ as above, we require the diagonal entry of $T_{j}$ to be nonzero, i.e.,

$$
\left(v_{2}^{(2)}\right)_{1}-\left(v_{1}^{(2)}\right)_{1} x_{1}=\left(v_{2}^{(2)}\right)_{1}-\frac{\left(v_{1}^{(2)}\right)_{1}\left(v_{2}^{(1)}\right)_{1}}{\left(v_{1}^{(1)}\right)_{1}} \neq 0
$$

in the case of $T_{2}$ and otherwise

$$
\left(v_{((j-1) \bmod 2)+1}^{(j)}\right)_{1} \neq 0 \quad \text { for } \quad j=3, \ldots, m .
$$

We observe that $S$ being invertible is a generic condition on $\left(v_{1}, v_{2}\right)$, i.e., the set $\Omega \subseteq$ $\mathbb{C}^{n} \times \mathbb{C}^{n}$, such that for all $\left(v_{1}, v_{2}\right) \in \Omega$ the conditions $\left(v_{1}^{(1)}\right)_{1} \neq 0,(2.13)$, and (2.14) hold, is generic. 
Let us now consider 2). If (2.5) is regular, it can be confirmed by straightforward computation that the vectors from (2.8) and (2.9) are Jordan chains of lengths $n_{4}, \ldots, n_{m}$ corresponding to $\hat{\lambda}$. Also, one validates that all vectors from (2.7)-(2.9) are linearly independent; it remains to consider (2.7) denoting the $j$ th given vector by $c_{j}$. In order to verify that $\left\{c_{1}, \ldots, c_{n_{3}}\right\}$ is indeed a Jordan chain of (2.5) corresponding to $\widehat{\lambda}$, we will check that the conditions in (2.1) are satisfied recalling the following: By hypothesis, $\widehat{\lambda} E-A$ has the form

$$
\left(-J_{n_{1}}(0)\right) \oplus \cdots \oplus\left(-J_{n_{m}}(0)\right) \oplus(\widehat{\lambda} \widetilde{E}-\widetilde{A}),
$$

whereas the form of the transformed perturbation $\left[\left(\widehat{\lambda} \beta_{1}-\alpha_{1}\right) w_{1},\left(\widehat{\lambda} \beta_{2}-\alpha_{2}\right) w_{2}\right] L$ is given by (2.6). Keeping this in mind, we compute that the first condition in (2.1) is satisfied:

$$
\begin{aligned}
& \left(\widehat{\lambda} E-A+\left[\left(\widehat{\lambda} \beta_{1}-\alpha_{1}\right) w_{1},\left(\widehat{\lambda} \beta_{2}-\alpha_{2}\right) w_{2}\right] L\right) c_{1} \\
= & \left(\widehat{\lambda} E-A+\left[\left(\widehat{\lambda} \beta_{1}-\alpha_{1}\right) w_{1},\left(\widehat{\lambda} \beta_{2}-\alpha_{2}\right) w_{2}\right] L\right)\left(e_{1}-e_{n_{1}+n_{2}+1}-x_{1} e_{n_{1}+1}\right) \\
= & \left(\widehat{\lambda} \beta_{1}-\alpha_{1}\right) w_{1}+x_{1}\left(\widehat{\lambda} \beta_{2}-\alpha_{2}\right) w_{2}-\left(\widehat{\lambda} \beta_{1}-\alpha_{1}\right) w_{1}-x_{1}\left(\widehat{\lambda} \beta_{2}-\alpha_{2}\right) w_{2}=0 .
\end{aligned}
$$

Then, it remains to show that also the second condition in (2.1) is satisfied for $j=2, \ldots, n_{3}$ using the same prerequisites as above:

$$
\begin{aligned}
& \left(\widehat{\lambda} E-A+\left[\left(\widehat{\lambda} \beta_{1}-\alpha_{1}\right) w_{1},\left(\widehat{\lambda} \beta_{2}-\alpha_{2}\right) w_{2}\right] L\right) c_{j} \\
= & \left(\widehat{\lambda} E-A+\left[\left(\widehat{\lambda} \beta_{1}-\alpha_{1}\right) w_{1},\left(\widehat{\lambda} \beta_{2}-\alpha_{2}\right) w_{2}\right] L\right)\left(e_{j}-e_{n_{1}+n_{2}+j}-\left[e_{n_{1}+j}, \ldots, e_{n_{1}+1}\right]\left[x_{1}, \ldots, x_{j}\right]^{T}\right) \\
= & -e_{j-1}+x_{j}\left(\widehat{\lambda} \beta_{2}-\alpha_{2}\right) w_{2}+e_{n_{1}+n_{2}+j-1}+\left[e_{n_{1}+j-1}, \ldots, e_{n_{1}+1}\right]\left[x_{1}, \ldots, x_{j-1}\right]^{T}-x_{j}\left(\widehat{\lambda} \beta_{2}-\alpha_{2}\right) w_{2} \\
= & -e_{j-1}-x_{j-1} \beta_{2} w_{2}+e_{n_{1}+n_{2}+j-1}+\left[e_{n_{1}+j-1}, \ldots, e_{n_{1}+1}\right]\left[x_{1}, \ldots, x_{j-1}\right]^{T}+x_{j-1} \beta_{2} w_{2} \\
= & -\left(E+\left[\beta_{1} w_{1}, \beta_{2} w_{2}\right] L\right)\left(e_{j-1}-e_{n_{1}+n_{2}+j-1}-\left[e_{n_{1}+j-1}, \ldots, e_{n_{1}+1}\right]\left[x_{1}, \ldots, x_{j-1}\right]^{T}\right) \\
= & -\left(E+\left[\beta_{1} w_{1}, \beta_{2} w_{2}\right] L\right) c_{j-1} .
\end{aligned}
$$

To illustrate the above theorem, let us regard an example.

Example 2.7 Consider the matrix pencil $(E, A)=\left(I_{9}, J_{3}(0) \oplus J_{2}(0) \oplus J_{2}(0) \oplus J_{2}(0)\right)$, i.e., we have $\widehat{\lambda}=0$ and $\left(n_{1}, n_{2}, n_{3}, n_{4}\right)=(3,2,2,2)$, and a perturbation $(\Delta E, \Delta A)=$ $\left(u_{1} v_{1}^{T}, u_{2} v_{2}^{T}\right)$, where

$$
v_{1}^{T}=\left[\begin{array}{lll|ll|ll|ll}
1 & 2 & 3 & 0 & 0 & 1 & 0 & 0 & 0
\end{array}\right] \quad \text { and } \quad v_{2}^{T}=\left[\begin{array}{lll|ll|ll|ll}
1 & -1 & -2 \mid 1 & 0 \mid 0 & 0 \mid & 0
\end{array}\right]
$$

and $u_{1}, u_{2} \in \mathbb{C}^{9}$ are arbitrary. Then, the generic conditions on $\left(v_{1}, v_{2}\right)$ from the proof of Theorem 2.6 are satisfied. Thus, setting $S=\left[\begin{array}{ccc}1 & 2 & 3 \\ 1 & 2 \\ & 1\end{array}\right] \oplus I_{6}$, the pencil $S(E+\Delta E, A+\Delta A) S^{-1}$ 
is in partial Brunovsky form as in Theorem 2.6 given by

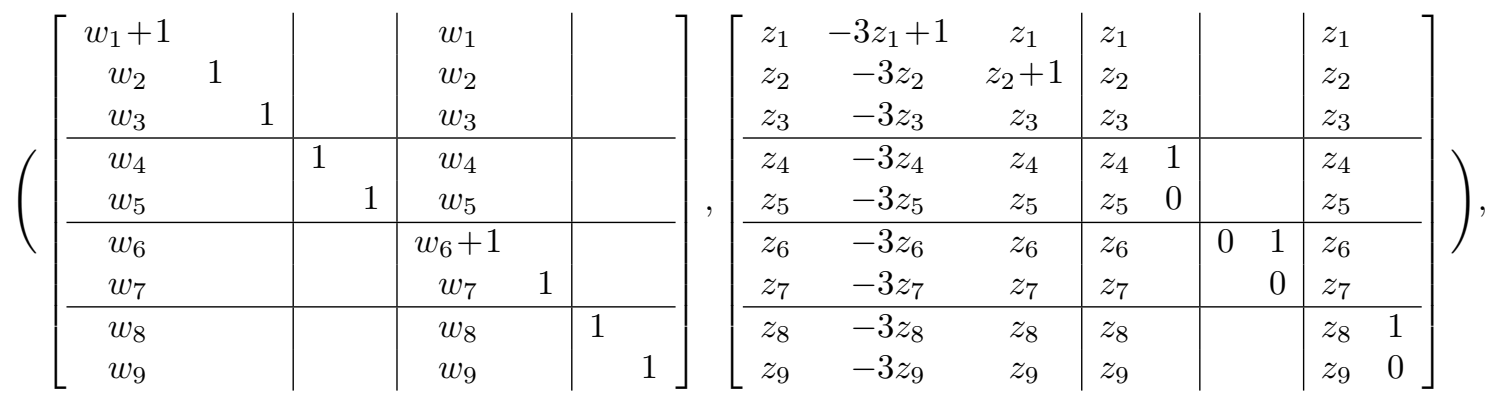

where $w=S u_{1}, z=S u_{2}$, and $x^{T}=[1,-3,1]$. The linearly independent chains of lengths $n_{3}$ and $n_{4}$ constructed in (2.7) and (2.8) are given by $e_{1}-e_{6}-e_{4}, e_{2}-e_{7}+3 e_{4}-e_{5}$ and $e_{4}-e_{8}, e_{5}-e_{9}$.

Before proceeding, we introduce the following phrase: A regular matrix pencil is said to have partial multiplicities greater than or equal to a certain list of multiplicities, e.g., $\left(n_{1}, \ldots, n_{k}\right)$, at some eigenvalue $\widehat{\lambda}$ if it has at least $k$ linearly independent Jordan chains at $\hat{\lambda}$ that have at least the lengths $n_{1}, \ldots, n_{k}$. We continue with a remark.

Remark 2.8 For any regular matrix pencil $(E, A) \in \mathbb{C}^{n, n} \times \mathbb{C}^{n, n}$ with partial multiplicities $n_{1} \geq \cdots \geq n_{m}>0$ at some eigenvalue $\widehat{\lambda}$, there exist invertible $V, W \in \mathbb{C}^{n, n}$ such that $V(E, A) W$ is in Weierstraß canonical form as in Theorem 2.2 with the $\widehat{\lambda}$ blocks coming first and ordered decreasingly with respect to their size. Then, if $(\Delta E, \Delta A)$ is a perturbation of rank 1 or 2 as in Theorem 2.4 or 2.6, the transformed perturbed pencil

$$
V(E, A) W+V(\Delta E, \Delta A) W
$$

can be transformed to partial Brunovsky form as in (2.3) or (2.5), depending on $(\Delta E, \Delta A)$ having rank 1 or 2 . Thus, if $(E+\Delta E, A+\Delta A)$ is regular, it generically has partial multiplicities greater than or equal to $\left(n_{2}, \ldots, n_{m}\right)$ or $\left(n_{3}, \ldots, n_{m}\right)$, respectively, at $\widehat{\lambda}$. We note that this lower bound on the block sizes of the perturbed pencil can also be obtained from $[4$, Lemma 2.1], but that the Theorems 2.4 and 2.6 will still be essential for constructing the desired Jordan chains.

We continue with the following Lemma.

Lemma 2.9 Let $(E, A) \in \mathbb{C}^{n, n} \times \mathbb{C}^{n, n}$ be regular and consider a perturbation of the form

$$
(\Delta E, \Delta A)=\left[\begin{array}{lll}
u_{1} & \ldots & u_{k}
\end{array}\right](\delta E, \delta A)\left[\begin{array}{lll}
u_{1} & \ldots & u_{k}
\end{array}\right]^{*},
$$

where $(\delta E, \delta A)$ is an arbitrary but fixed $k \times k$ pencil. Then, the following statements hold:

1) There exists a generic set $\Lambda \subseteq\left(\mathbb{C}^{n}\right)^{k}$, so that the perturbed pencil $(E+\Delta E, A+\Delta A)$ is regular for all $\left(u_{1}, \ldots, u_{k}\right) \in \Lambda$. 
2) Let there exist a generic set $\Lambda^{\prime} \subseteq\left(\mathbb{C}^{n}\right)^{k}$ such that $(E+\Delta E, A+\Delta A)$ has at least the algebraic multiplicity a at some $\widehat{\widehat{\lambda}} \in \mathbb{C}$ for all $\left(u_{1}, \ldots, u_{k}\right) \in \Lambda^{\prime}$. If $(E+\Delta E, A+\Delta A)$ is regular and has the algebraic multiplicity equal to a at $\widehat{\lambda}$ for one $\left(u_{1}, \ldots, u_{k}\right) \in\left(\mathbb{C}^{n}\right)^{k}$, this also holds on some generic subset of $\left(\mathbb{C}^{n}\right)^{k}$.

This Lemma is identical to [2, Lemma 2.4] except for the transpose ' $T$ ' instead of the conjugate transpose ' $*$ ' in equation (2.15), but since the proof is analogous in both cases, it will be omitted. Our final tool for examining the effects of perturbations is the following set of inequalities. For all matrix pencils $(E, A),(\Delta E, \Delta A) \in \mathbb{C}^{n, n} \times \mathbb{C}^{n, n}$ we have by $[4$, Section 1]:

$$
\begin{aligned}
\operatorname{rank}(\widehat{\lambda} E-A)-\operatorname{rank}(\widehat{\lambda} \Delta E-\Delta A) & \leq \operatorname{rank}(\widehat{\lambda}(E+\Delta E)-(A+\Delta A)) \\
& \leq \operatorname{rank}(\widehat{\lambda} E-A)+\operatorname{rank}(\widehat{\lambda} \Delta E-\Delta A)
\end{aligned}
$$

for any $\widehat{\lambda} \in \mathbb{C}$. Therefore, if $(E, A)$ and $(E+\Delta E, A+\Delta A)$ are both regular, the geometric multiplicity of $(E, A)$ at an eigenvalue $\hat{\lambda}$ cannot change by more than $\operatorname{rank}(\widehat{\lambda} \Delta E-\Delta A)$ under perturbation. Note that only the rank of the matrix $\hat{\lambda} \Delta E-\Delta A$ matters for this estimate and that this number can be zero even for nonzero perturbations.

\subsection{Hermitian Kronecker canonical form and sign characteristic}

The following Kronecker-like canonical form for Hermitian matrix pencils was deduced in [12] for arbitrary Hermitian matrix pencils; even though we only consider the regular case here. We remind the reader that $R_{n}$ denotes the $n \times n$ reverse identity matrix.

Theorem 2.10 (Hermitian Kronecker form) Let $(E, A) \in \mathbb{C}^{n, n} \times \mathbb{C}^{n, n}$ be a regular Hermitian matrix pencil. Then, there is a nonsingular matrix $X \in \mathbb{C}^{n, n}$, such that

$$
\begin{aligned}
X(E, A) X^{*}= & \left(\bigoplus_{i=1}^{p} \sigma_{i}\left(R_{\gamma_{i}}, R_{\gamma_{i}} J_{\gamma_{i}}\left(\lambda_{i}\right)\right)\right) \oplus\left(\bigoplus_{j=1}^{q} \eta_{j}\left(R_{\delta_{j}} J_{\delta_{j}}(0), R_{\delta_{j}}\right)\right) \oplus \\
& \left(\bigoplus_{k=1}^{r}\left(\left[\begin{array}{cc}
0 & R_{\phi_{k}} \\
R_{\phi_{k}} & 0
\end{array}\right],\left[\begin{array}{cc}
0 & R_{\phi_{k}} J_{\phi_{k}}\left(\mu_{k}\right) \\
R_{\phi_{k}} J_{\phi_{k}}\left(\overline{\mu_{k}}\right) & 0
\end{array}\right]\right)\right)
\end{aligned}
$$

where $\lambda_{i} \in \mathbb{R}$ for $i=1, \ldots, p, \mu_{k} \in \mathbb{C} \backslash \mathbb{R}$ for $k=1, \ldots, r$, and all $\sigma_{i}$ and $\eta_{j}$ are signs, i.e., either -1 or +1 .

Hereby, the entirety of the signs corresponding to Jordan blocks associated with some real (or infinite) eigenvalue $\widehat{\lambda}$ is called the sign characteristic of $(E, A)$ at $\widehat{\lambda}$. Based on the above canonical form, we can characterize the sign characteristic of Hermitian pencils analogously to the sign characteristic of $H$-selfadjoint matrices in [9]. We let $\widehat{\lambda} \in \mathbb{R}$ be a fixed eigenvalue of $(E, A)$ and $\Psi_{1} \subseteq \mathbb{C}^{n}$ be its eigenspace at $\widehat{\lambda}$. For $x \in \Psi_{1} \backslash\{0\}$, denote by 
$\nu(x)$ the maximal length of a Jordan chain of $(E, A)$ beginning with the eigenvector $x$ and define $\Psi_{s}:=\left\{x \in \Psi_{1} \mid x=0\right.$ or $\left.\nu(x) \geq s\right\}$. Setting $\gamma:=\max \left\{\nu(x) \mid x \in \Psi_{1} \backslash\{0\}\right\}$, it is

$$
\operatorname{Ker}(\widehat{\lambda} E-A)=\Psi_{1} \supseteq \Psi_{2} \supseteq \cdots \supseteq \Psi_{\gamma} \supseteq \Psi_{\gamma+1}=\{0\}
$$

and the following theorem is obtained parallel to [9, Theorem 5.8.1], to which we refer the reader for the proof.

Theorem 2.11 Let $(E, A) \in \mathbb{C}^{n, n} \times \mathbb{C}^{n, n}$ be regular and Hermitian and $\widehat{\lambda} \in \mathbb{R}$ an eigenvalue. For $s=1, \ldots, \gamma$ let

$$
f_{s}(x, y)=x^{*} E y^{(s)} ; \quad x \in \Psi_{s}, \quad y \in \Psi_{s} \backslash\{0\},
$$

where $y=y^{(1)}, y^{(2)}, \ldots, y^{(s)}$ is a Jordan chain of $(E, A)$ corresponding to $\hat{\lambda}$ with eigenvector y. Letting $f_{s}(x, 0)=0$, then:

(i) $f_{s}(x, y)$ does not depend on the choice of $y^{(2)}, \ldots, y^{(s)}$.

(ii) There is a selfadjoint linear transformation $G_{s}: \Psi_{s} \rightarrow \Psi_{s}$ with

$$
f_{s}(x, y)=x^{*} G_{s} y ; \quad x, y \in \Psi_{s} .
$$

(iii) For this $G_{s}$, we have $\operatorname{Ker} G_{s}=\Psi_{s+1}$.

(iv) The number of positive (or negative) eigenvalues of $G_{s}$, counting multiplicities, coincides with the number of positive (or negative, respectively) signs in the sign characteristic of $(E, A)$ associated with Jordan blocks of size $s$ at $\widehat{\lambda}$.

By this theorem, the sign characteristic of a Hermitian matrix pencil can be described as the number of positive and negative eigenvalue of some selfadjoint linear map. Therefore, it will be cruicial how the number of positive and negative eigenvalues of an Hermitian matrix is altered under rank-1 perturbations. The following lemma is obtained by applying wellknown results on the eigenvalues of Hermitian matrices and extracted from the proof of [15, Theorem 3.3].

Lemma 2.12 Let $A \in \mathbb{C}^{n, n}$ be Hermitian and invertible with the eigenvalues $\lambda_{1}, \ldots, \lambda_{n}$. Then, for any $\lambda_{n+1} \in \mathbb{R}$ and $u \in \mathbb{C}^{n}$, so that $A+\lambda_{n+1} u u^{*}$ is invertible, the signs of its eigenvalues are obtained by removing either exactly one sign -1 or exactly one sign +1 from the list $\left\{\operatorname{sgn}\left(\lambda_{1}\right), \ldots, \operatorname{sgn}\left(\lambda_{n+1}\right)\right\}$.

Proof. Let us assume that $\lambda_{1} \leq \cdots \leq \lambda_{k}<0<\lambda_{k+1} \leq \cdots \leq \lambda_{n}$ for some $k \in\{0,1, \ldots, n\}$ and that $\lambda_{n+1}>0$ (in the case $\lambda_{n+1}=0$ there is nothing to show). Further, letting $\widetilde{\lambda}_{1} \leq \cdots \leq \widetilde{\lambda}_{n}$ be the eigenvalues of $A+\lambda_{n+1} u u^{*}$, by [11, Corollary 4.3.3] we have $\lambda_{j} \leq \widetilde{\lambda}_{j}$ for $j=1, \ldots, n$; in particular $A+\lambda_{n+1} u u^{*}$ has at least $n-k$ positive eigenvalues. Now, from [11, Theorem 4.3.4] we obtain

$$
\lambda_{j} \leq \widetilde{\lambda}_{j+1} \leq \lambda_{j+2}, \quad j=1,2, \ldots, n-2,
$$

which in particular yields $\widetilde{\lambda}_{k-1} \leq \lambda_{k}<0$, i.e, $A+\lambda_{n+1} u u^{*}$ hat at least $k-1$ negative eigenvalues. Since $A+\lambda_{n+1} u u^{*}$ is invertible, its $k$ th eigenvalue can only have sign $+1=$ $\operatorname{sgn}\left(\lambda_{n+1}\right)$ or $\operatorname{sign}-1=\operatorname{sgn}\left(\lambda_{k}\right)$. 


\subsection{Properties of Hermitian perturbations}

It is our motivation to consider Hermitian rank- $k$ perturbations of the form

$$
\left[\begin{array}{lll}
\widetilde{u}_{1} & \ldots & \widetilde{u}_{k}
\end{array}\right](\delta E, \delta A)\left[\begin{array}{lll}
\widetilde{u}_{1} & \ldots & \widetilde{u}_{k}
\end{array}\right]^{*},
$$

where $(\delta E, \delta A)$ is a generic Hermitian $k \times k$ pencil and $\widetilde{u}_{1}, \ldots, \widetilde{u}_{k} \in \mathbb{C}^{n}$ are certain generic vectors. Since the set of Hermitian $k \times k$ pencils

$$
\mathcal{H}_{k}:=\left\{(E, A) \in \mathbb{C}^{k, k} \times \mathbb{C}^{k, k} \mid(E, A) \text { is Hermitian }\right\}
$$

is an $\mathbb{R}$-vector space of dimension $2 k^{2}$, we consider a subset of $\mathcal{H}_{k}$ to be generic if it can canonically be identified with a generic subset of $\mathbb{R}^{2 k^{2}}$. Hence, we denote with $[(E, A)]_{\mathcal{B}} \in$ $\mathbb{R}^{2 k^{2}}$ the coordinates of the Hermitian matrix pencil $(E, A)$ with respect to an $\mathbb{R}$-basis $\mathcal{B}$ of $\mathcal{H}_{k}$. Then, the following lemma holds.

Lemma 2.13 The set $\Gamma$ of regular Hermitian matrix pencils with distinct eigenvalues is a generic subset of $\mathcal{H}_{k}$.

Proof. We follow the procedure laid out in the proof of [3, Theorem 2.3]. Let $(E, A) \in \mathcal{H}_{k}$ and consider its characteristic polynomial $\sum_{j=0}^{k} c_{j} \lambda^{j}:=\chi_{(E, A)}(\lambda)=\operatorname{det}(\lambda E-A)$. We observe that the coefficients $c_{j}=c_{j}(E, A)$ for $j=0,1, \ldots, n$ depend polynomially on the coordinates $[(E, A)]_{\mathcal{B}}$ and that $c_{j}(E, A) \neq 0$ for at least one $j$ if $(E, A)$ is regular.

Recall that the Sylvester resultant matrix of two polynomials $s(\lambda)$ and $t(\lambda)$, denoted by $S(s(\lambda), t(\lambda))$, is a square matrix of dimension $\operatorname{deg}(s)+\operatorname{deg}(t)$. It is well-known that its entries are coefficients of $s(\lambda)$ and $t(\lambda)$ and that the rank defect of $S(s(\lambda), t(\lambda))$ is exactly the degree of the greatest common divisor of $s(\lambda)$ and $t(\lambda)$ (see, e.g., [13]). We define

$$
p(E, A):=\operatorname{det} S\left(\chi_{(E, A)}(\lambda), \frac{\partial \chi_{(E, A)}(\lambda)}{\partial \lambda}\right) \quad \text { and } \quad q(E, A):=\operatorname{det} S\left(\chi_{(E, A)}(\lambda), \frac{\partial \chi_{(E, A)}(\lambda)}{\partial \lambda}\right)
$$

and observe that $p(E, A)$ and $q(E, A)$ both depend polynomially on the coordinates $[(E, A)]_{\mathcal{B}}$ and that the pencil $(E, A)$ does not have multiple eigenvalues (neither finite nor infinite) if and only if $p(E, A) q(E, A) \neq 0$. Clearly, $p(E, A) q(E, A)$ is not constantly zero since there exist regular Hermitian pencils with distinct eigenvalues, so that the set

$$
\Gamma:=\left\{(E, A) \in \mathcal{H}_{k} \mid p(E, A) q(E, A) \neq 0 \text { and } \exists j \in\{0,1, \ldots, k\} \text { with } c_{j}(E, A) \neq 0\right\}
$$

of regular Hermitian matrix pencils with distinct eigenvalues is a generic subset of $\mathcal{H}_{k}$.

Now, for $(\delta E, \delta A)$ in (2.17) there exists an invertible $X \in \mathbb{C}^{k, k}$ such that $X(\delta E, \delta A) X^{*}$ is in Hermitian Kronecker form as in Theorem 2.10; thus (2.17) can be transformed to

$$
\left[\begin{array}{lll}
u_{1} & \ldots & u_{k}
\end{array}\right] X(\delta E, \delta A) X^{*}\left[\begin{array}{lll}
u_{1} & \ldots & u_{k}
\end{array}\right]^{*}
$$

setting $u_{j}=\widetilde{u}_{j} X^{-1}$ for $j=1, \ldots, k$. Hereby, considering all $\left[\widetilde{u}_{1}, \ldots, \widetilde{u}_{k}\right]$ that are elements of some generic subset of $\mathbb{C}^{n, k}$ is equivalent to considering all $\left[u_{1}, \ldots, u_{k}\right]$ that are elements of some generic subset of $\mathbb{C}^{n, k}$, since the respective generic sets can be transformed into one another by multiplication with an invertible matrix. Additionally, assuming $(\delta E, \delta A) \in \Gamma$ with $\Gamma$ as in Lemma 2.13, then $X(\delta E, \delta A) X^{*}$ can only consist of the following types of blocks: 
- $[\lambda \beta-\alpha]-$ a $1 \times 1$ block corresponding to the real eigenvalue $\alpha / \beta$ with the sign $\operatorname{sgn}(\beta)$ (and corresponding to $\infty$ with $\operatorname{sign} \operatorname{sgn}(\alpha)$ if $\beta=0$ ),

- $\left[{ }_{\lambda-\bar{\mu}}{ }^{\lambda-\mu}\right]-$ two paired $1 \times 1$ blocks corresponding to the eigenvalues $\mu, \bar{\mu} \in \mathbb{C} \backslash \mathbb{R}$,

as all other regular blocks in Hermitian Kronecker form have multiple eigenvalues. Therefore, the perturbation (2.17) is the sum of, on the one hand, rank-1 perturbations of the form

$$
(\lambda \beta-\alpha) u u^{*},
$$

that we study in Section 3, and on the other hand of rank-2 perturbations of the form

$$
\left[\begin{array}{ll}
u & v
\end{array}\right]\left[\begin{array}{cc}
0 & \lambda-\mu \\
\lambda-\bar{\mu} & 0
\end{array}\right]\left[\begin{array}{l}
u^{*} \\
v^{*}
\end{array}\right]
$$

subject of Section 4 .

\section{Hermitian rank-1 perturbations}

We will now turn to our main results, remarking that since the potential infinite eigenvalue of a Hermitian pencil $(E, A)$ is the zero eigenvalue of the reverse pencil $(A, E)$, it is sufficient to state these theorems in terms of the finite eigenvalues of $(E, A)$.

In this section, we consider rank-1 perturbations of the form (2.18) with $\alpha, \beta \in \mathbb{R}$, since otherwise the perturbation were not Hermitian. In the next two theorems, we will characterize the generic canonical form of regular Hermitian matrix pencils under rank-1 perturbation as follows: In Theorem 3.1 we will analyze the Jordan structure (i.e., the sizes of the Jordan blocks) and in Theorem 3.3 the sign characteristic will be determined under perturbation.

Theorem 3.1 Let $(E, A) \in \mathbb{C}^{n, n} \times \mathbb{C}^{n, n}$ be regular and Hermitian with the partial multiplicities $n_{1} \geq \cdots \geq n_{m}>0$ associated with some eigenvalue $\hat{\lambda} \in \mathbb{C}$. Then, for each $(\alpha, \beta) \in(\mathbb{R} \times \mathbb{R}) \backslash\{0\}$ there exists a generic set $\Omega_{\alpha, \beta}^{\prime} \subseteq \mathbb{C}^{n}$, such that for all $u \in \Omega_{\alpha, \beta}^{\prime}$, $\left(E+\beta u u^{*}, A+\alpha u u^{*}\right)$ is regular and has the partial multiplicities $\left(n_{2}, \ldots, n_{m}\right)$ if $\widehat{\lambda} \beta \neq \alpha$ and $\left(n_{2}, \ldots, n_{m}, 1\right)$ otherwise at $\widehat{\lambda}$.

Proof. Because of Theorem 2.4 (cf. Remark 2.8) and (2.16), the perturbed pencil ( $E+$ $\left.\beta u u^{*}, A+\alpha u u^{*}\right)$ generically has partial multiplicities greater than or equal to the above given multiplicities in each case. In view of Lemma 2.9, it will be sufficient to present one particular perturbation in each case that creates these partial multiplicities to conclude the proof. We assume that $(E, A)$ is in Hermitian Kronecker form as in Theorem 2.10 and that the blocks corresponding to $\widehat{\lambda}$ are coming first and in nonincreasing order with respect to their size. 
Case $\hat{\lambda} \in \mathbb{C} \backslash \mathbb{R}$. Consider the first block of $(E, A)$ corresponding to $\hat{\lambda}$ (and the paired complex conjugate block) and set $u:=e_{1}+e_{n_{1}+1}$. Then the first two blocks of $\left(E+\beta u u^{*}, A+\alpha u u^{*}\right)$ are given by

$$
\left[\begin{array}{cc}
(\lambda \beta-\alpha) e_{1} e_{1}^{T} & -R_{n_{1}} J_{n_{1}}(\widehat{\lambda}-\lambda)+(\lambda \beta-\alpha) e_{1} e_{1}^{T} \\
-R_{n_{1}} J_{n_{1}}(\overline{\widehat{\lambda}}-\lambda)+(\lambda \beta-\alpha) e_{1} e_{1}^{T} & (\lambda \beta-\alpha) e_{1} e_{1}^{T}
\end{array}\right]
$$

clearly having full rank at $\hat{\lambda}$. Since all other blocks are unchanged, this particular perturbation clearly creates the partial multiplicities $\left(n_{2}, \ldots, n_{m}\right)$ at $\hat{\lambda}$.

Case $\hat{\lambda} \in \mathbb{R}$. Consider the first block of $(E, A)$ at $\hat{\lambda}$ having the sign $\epsilon \in\{ \pm 1\}$ and set $u:=e_{1}$. Then, the first block of $\left(E+\beta u u^{*}, A+\alpha u u^{*}\right)$ is given by

$$
-\epsilon R_{n_{1}} J_{n_{1}}(\widehat{\lambda}-\lambda)+(\lambda \beta-\alpha) e_{1} e_{1}^{T}
$$

not having the eigenvalue $\widehat{\lambda}$ if $\beta \widehat{\lambda} \neq \alpha$ and having the simple eigenvalue $\widehat{\lambda}$ if $\beta \widehat{\lambda}=\alpha$, which creates the desired multiplicities as no other blocks are perturbed.

This theorem shows that the generic Jordan structure of regular Hermitian matrix pencils under Hermitian rank-1 perturbations is the same as under unstructured rank-1 perturbations, cf. [3, Theorem 2.10] or [4, Theorem 3.3]. However, in the case of Hermitian perturbations, the perturbed pencil still has a sign characteristic associated with its real eigenvalues that we will analyze in the following. Let us first consider an example.

Example 3.2 The Hermitian $4 \times 4$ matrix pencil

$$
(E, A)=\left(\left[\begin{array}{cccc}
0 & 1 & & \\
1 & 0 & & \\
& & 0 & -1 \\
& & -1 & 0
\end{array}\right],\left[\begin{array}{cccc}
0 & 0 & & \\
0 & 1 & & \\
& & 0 & 0 \\
& & 0 & -1
\end{array}\right]\right) \in \mathbb{C}^{4,4} \times \mathbb{C}^{4,4}
$$

clearly consists of two Jordan blocks of size two corresponding to 0, where the first one has sign +1 and the second one has sign -1 . We consider a Hermitian rank-1 perturbation of the form $\left(\beta u u^{*}, 0\right)$, where $\beta$ is a real parameter and $u=\left[u_{1}, u_{2}, u_{3}, u_{4}\right]^{T} \in \mathbb{C}^{4}$. By Theorem 3.1 the perturbed pencil $\left(E+\beta u u^{*}, A\right)$ is generically (with respect to $u$ ) regular and has two linearly independent Jordan chains of lengths two and one at 0.

To extract the 2 signs of the perturbed pencil, we need to construct these Jordan chains. Clearly, $E(E, A)$ is in Weierstraß canonical form and whenever the generic condition $u_{1}, u_{3} \neq 0$ is satisfied, the matrix $S:=\operatorname{Toep}\left(\bar{u}_{1}, \bar{u}_{2}\right) \oplus \operatorname{Toep}\left(\bar{u}_{3}, \bar{u}_{4}\right)$ is invertible; hence the pencil $S E\left(E+\beta u u^{*}, A\right) S^{-1}$ is in partial Brunovsky form as in Theorem 2.4, i.e., $u^{*} S^{-1}=\left[e_{1,2}^{T}, e_{1,2}^{T}\right]$. Thus, its chain of length two is given by $e_{1}-e_{3}, e_{2}-e_{4}$ and also, the linearly independent chain of length one is $e_{1}$. Now, we consider the matrix pencil

$$
S^{-*}\left(E+\beta u u^{*}, A\right) S^{-1},
$$

that has the Jordan chains given above (left-multiplication with an invertible matrix does not change the Jordan chains of a matrix pencil) and also the sign characteristic of $(E+$ 
$\left.\beta u u^{*}, A\right)$ since it is perserved under $*$-congruence transformations. To obtain the sign characteristic of (3.1), the matrix

$$
F:=S^{-*}\left(E+\beta u u^{*}\right) S^{-1}=\left[\begin{array}{cc|cc}
\beta & 1 /\left|u_{1}\right|^{2} & \beta & 0 \\
1 /\left|u_{1}\right|^{2} & * & 0 & 0 \\
\hline \beta & 0 & \beta & -1 /\left|u_{3}\right|^{2} \\
0 & 0 & -1\left|u_{3}\right|^{2} & *
\end{array}\right]
$$

is crucial: By Theorem 2.11, the sign corresponding to the block of size one of (3.1) is given by the sign of (the eigenvalue of) $e_{1}^{T} F e_{1}=\beta$, i.e., the sign that is attached to the eigenvalue 0 in the perturbation $\left(\beta u u^{*}, 0\right)$ and the sign attached to the block of size two of (3.1) is the sign of

$$
\left(e_{1}-e_{3}\right)^{T} F\left(e_{2}-e_{4}\right)=\frac{1}{\left|u_{1}\right|^{2}}-\frac{1}{\left|u_{3}\right|^{2}} .
$$

In particular, this sign cannot generically be determined to be +1 or -1 .

Now, we turn to the general case employing similar methods. Thereby, let us group together Jordan blocks of the same size, i.e.,

$$
\left(n_{1}, n_{2}, \ldots, n_{m}\right)=(\underbrace{s_{1}, \ldots, s_{1}}_{t_{1}}, \ldots, \underbrace{s_{\nu}, \ldots, s_{\nu}}_{t_{\nu}})
$$

where $s_{1}>s_{2}>\cdots>s_{\nu}>0$. Then, for $s=1,2, \ldots$ we denote by $\mathcal{L}_{s}$ the list of signs associated with blocks of size $s$; if $(E, A)$ does not have a block of size $s$, let $\mathcal{L}_{s}$ be the empty list. Using this convention, we achieve a concise phrasing of the following theorem. Recall that $\Omega_{\alpha, \beta}^{\prime}$ denotes the generic set from Theorem 3.1.

Theorem 3.3 Let $(E, A) \in \mathbb{C}^{n, n} \times \mathbb{C}^{n, n}$ be regular and Hermitian with the partial multiplicities (3.2) and let the list of signs $\mathcal{L}_{s}$ be attached to its blocks of size $s=1,2, \ldots$ at the eigenvalue $\hat{\lambda} \in \mathbb{R}$. Then, for each $(\alpha, \beta) \in(\mathbb{R} \times \mathbb{R}) \backslash\{0\}$ there exists a generic set $\Omega_{\alpha, \beta} \subseteq \Omega_{\alpha, \beta}^{\prime}$, such that for all $u \in \Omega_{\alpha, \beta}$, the list of signs $\mathcal{L}_{s}^{\prime \prime}$ attached to the blocks of size $s$ of $\left(E+\beta u u^{*}, A+\alpha u u^{*}\right)$ at $\widehat{\lambda}$ is obtained by subsequently executing the following steps:

- If $s=1$ and $\hat{\lambda} \beta=\alpha$, then $\mathcal{L}_{s}^{\prime}$ is obtained from $\mathcal{L}_{s}$ by adding $\operatorname{sgn}(\beta)$, else $\mathcal{L}_{s}^{\prime}:=\mathcal{L}_{s}$.

- If $s=s_{1}$, then $\mathcal{L}_{s}^{\prime \prime}$ is obtained from $\mathcal{L}_{s}^{\prime}$ by removing either exactly one sign -1 or exactly one sign +1 , else $\mathcal{L}_{s}^{\prime \prime}:=\mathcal{L}_{s}^{\prime}$.

Proof. We assume $(E, A)$ to be in Hermitian Kronecker form as in Theorem 2.10 with the $\widehat{\lambda}$ blocks coming first and ordered by their size. Left-multiplying this matrix pencil with $E$, we obtain that the first blocks of $E(E, A)$ are in Weierstraß canonical form. In order to 
transform the pencil $E\left(E+\beta u u^{*}, A+\alpha u u^{*}\right)$ to partial Brunovsky form as in Theorem 2.4, let us partition $u$ as follows

$$
u=\left[\begin{array}{c}
u^{(1)} \\
\vdots \\
u^{(\nu)} \\
\widetilde{u}
\end{array}\right], \quad u^{(i)}=\left[\begin{array}{c}
u^{(i, 1)} \\
\vdots \\
u^{\left(i, t_{i}\right)}
\end{array}\right], \quad u^{(i, j)}=\left[\begin{array}{c}
u_{1}^{(i, j)} \\
\vdots \\
u_{s_{i}}^{(i, j)}
\end{array}\right] \in \mathbb{C}^{s_{i}}, \quad j=1, \ldots, t_{i}, \quad i=1, \ldots, \nu .
$$

Assuming the generic condition $u_{1}^{(i, j)} \neq 0$ to be satisfied for all $i, j$, the matrix $S$ from Remark 2.5 is invertible and given by

$$
S:=\left(\bigoplus_{i=1}^{\nu} \bigoplus_{j=1}^{t_{i}} \operatorname{Toep}\left(\overline{u^{(i, j)}}\right)\right) \oplus I_{n-a}
$$

where $a:=s_{1} t_{1}+\cdots+s_{\nu} t_{\nu}$. Since for this $S$ holds

$$
u^{*} S^{-1}=[\underbrace{e_{1, s_{1}}^{T} \ldots}_{t_{1}} e_{1, s_{1}}^{T} \cdots \underbrace{e_{1, s_{\nu}}^{T} \ldots}_{t_{\nu}} e_{1, s_{\nu}}^{T} \tilde{u}^{*}]=: e^{*},
$$

clearly $S E\left(E+\beta u u^{*}, A+\alpha u u^{*}\right) S^{-1}$ is in partial Brunovsky form as in Theorem 2.4 and thus has the Jordan chains in (2.4) associated with its eigenvalue $\widehat{\lambda}$. Then, the matrix pencil

$$
S^{-*}\left(E+\beta u u^{*}, A+\alpha u u^{*}\right) S^{-1}
$$

also has these chains and additionally, it has the same sign characteristic as $\left(E+\beta u u^{*}, A+\right.$ $\left.\alpha u u^{*}\right)$. To extract this sign characteristic of (3.4), we proceed similarly to the proof of $[15$, Theorem 3.3]. Of great importance will be the matrix

$$
F:=F_{1}+F_{2}:=S^{-*} E S^{-1}+\beta S^{-*} u u^{*} S^{-1} .
$$

Letting $\mathcal{L}_{s_{i}}=\left\{\epsilon_{i, 1}, \ldots, \epsilon_{i, t_{i}}\right\}$ for $i=1, \ldots, \nu$, the topleft $a \times a$ block of $F_{1}$ is given by

$$
\bigoplus_{i=1}^{\nu} \bigoplus_{j=1}^{t_{i}} T_{i, j}, \quad \text { where } \quad T_{i, j}=\left[\begin{array}{ccc}
0 & & \frac{\epsilon_{i, j}}{\left|u_{1}^{(i, j)}\right|^{2}} \\
& . \cdot & \vdots \\
\frac{\epsilon_{i, j}}{\left|u_{1}^{i, j}\right|^{2}} & \cdots & *
\end{array}\right] \in \mathbb{C}^{s_{i}, s_{i}}
$$

and by (3.3), clearly $F_{2}=\beta e e^{*}$. Now, by Theorem 2.11 the sign characteristic of (3.4) at blocks of size $s$ at $\hat{\lambda}$ is given by the signs of the eigenvalues of some selfadjoint linear map $G_{s}: \Psi_{s} \rightarrow \Psi_{s}$, where we have

$$
\begin{aligned}
& \Psi_{s}=\left\{x \in \mathbb{C}^{n} \backslash\{0\} \mid\right. \hat{\lambda}\left(E+\beta u u^{*}\right) x=\left(A+\alpha u u^{*}\right) x \text { and } x \text { can be extended to } \\
&\text { a chain of } \left.\left(E+\beta u u^{*}, A+\alpha u u^{*}\right) \text { of at least length } s\right\} \cup\{0\} .
\end{aligned}
$$


We will obtain these signs by computing the inertia of a matrix representation of the map

$$
f_{s}: \Psi_{s} \times \Psi_{s} \rightarrow \mathbb{C}, \quad(x, y) \mapsto x^{*} G_{s} y=x^{*} F y^{(s)}
$$

with respect to a suitable basis of $\Psi_{s}$. To construct this basis of $\Psi_{s}$, recall that of the Jordan chains of (3.4) from (2.4), the following ones have length $s_{i}$ :

$$
x_{i, k}^{(j)}:=e_{j}-e_{\kappa_{i}+(k-1) s_{i}+j}, \quad j=1, \ldots, s_{i}, \quad \begin{array}{ll}
k=2, \ldots, t_{1} & \text { if } i=1, \\
k=1, \ldots, t_{i} & \text { else }
\end{array}
$$

whereby $\kappa_{i}:=t_{1} s_{1}+\cdots+s_{i-1} t_{i-1}$, i.e., for each pair of indices $(i, k)$ there is the Jordan chain $x_{i, k}^{(1)}, \ldots, x_{i, k}^{\left(s_{i}\right)}$. In the remainder of this paper, we will usually distinguish the vectors of one chain by their superscript and for brevity omit the superscript if equal to one, e.g., $x_{i, k}:=x_{i, k}^{(1)}$ in (3.6). Also, in the case $\beta \widehat{\lambda}=\alpha$, there exists one more chain of (3.4) that has exactly length one (cf. Theorem 3.1) and is linearly independent from all chains in (3.6). It is straightforward to verify that this chain always consists of the first standard basis vector $e_{1}$ (recall that in this case the perturbation $(\lambda \beta-\alpha) u u^{*}$ is equal to 0 at $\widehat{\lambda}$ ).

Since under a rank-1 perturbation of the matrix pencil $(E, A)$ by Theorem 3.1 generically one block of size $s_{1}$ is destroyed and one block of size one is created if $\beta \widehat{\lambda}=\alpha$, we consider the following (mutually exclusive) classes of Jordan blocks:

(i) blocks of size $s_{i}<s_{1}$ if either $s_{i}>1$ or $\beta \widehat{\lambda} \neq \alpha$,

(ii) blocks of size $1<s_{1}$ if $\beta \widehat{\lambda}=\alpha$,

(iii) blocks of size $s_{1}$ if either $s_{1}>1$ or $\beta \widehat{\lambda} \neq \alpha$,

(iv) blocks of size $1=s_{1}$ if $\beta \widehat{\lambda}=\alpha$.

Blocks of type (i): To extract the signs of the nonzero eigenvalues of a matrix representation of $f_{s_{i}}$, we consider a basis of $\Psi_{s_{i}}$, whose last $\operatorname{dim}\left(\Psi_{s_{i}+1}\right)$ vectors form a basis of $\Psi_{s_{i}+1}$. Since by Theorem 3.1 the pencil (3.4) generically has $t_{i}$ linearly independent Jordan chains of length $s_{i}$ at $\widehat{\lambda}$, the first $t_{i}=\operatorname{dim}\left(\Psi_{s_{i}+1}\right)-\operatorname{dim}\left(\Psi_{s_{i}}\right)$ vectors of this basis can be chosen as $x_{i, 1}, \ldots, x_{i, t_{i}}$ as in (3.6). Then, as basis vectors in $\Psi_{s_{i}+1}$ lie in $\operatorname{Ker} G_{s_{i}}$, we do not need to consider them since they correspond to the zero part of the matrix representation of $f_{s_{i}}$.

Thus, it remains to compute $f_{s_{i}}\left(x_{i, k}, x_{i, \ell}\right)=x_{i, k}^{*} F x_{i, \ell}^{\left(s_{i}\right)}$ for $k, \ell=1, \ldots, t_{i}$. From (3.5) we observe that the first needed term is $x_{i, k}^{*} F_{1} x_{i, \ell}^{\left(s_{i}\right)}$, which is given as follows:

$$
\underbrace{e_{1}^{*} F_{1} e_{s_{i}}}_{=0}-\underbrace{e_{1}^{*} F_{1} e_{\kappa_{i}+\ell s_{i}}}_{=0}-\underbrace{e_{\kappa_{i}+(k-1) s_{i}+1}^{*} F_{1} e_{s_{i}}}_{=0}+e_{\kappa_{i}+(k-1) s_{i}+1}^{*} F_{1} e_{\kappa_{i}+\ell s_{i}}= \begin{cases}\frac{\epsilon_{i, k}}{\left|u_{1}^{i, k}\right|^{2}} & \text { if } k=\ell, \\ 0 & \text { if } k \neq \ell,\end{cases}
$$

where the first terms were simplified using $s_{i}<s_{1}$. Then, the second term $x_{i, k}^{*} F_{2} x_{i, \ell}^{\left(s_{i}\right)}$ is given by

$$
\underbrace{e_{1}^{*} F_{2} e_{s_{i}}}_{=\beta \delta_{1, s_{i}}}-\underbrace{e_{1}^{*} F_{2} e_{\kappa_{i}+\ell s_{i}}}_{=\beta \delta_{1, s_{i}}}-\underbrace{e_{\kappa_{i}+(k-1) s_{i}+1}^{*} F_{2} e_{s_{i}}}_{=\beta \delta_{1, s_{i}}}+\underbrace{e_{\kappa_{i}+(k-1) s_{i}+1}^{*} F_{2} e_{\kappa_{i}+\ell s_{i}}}_{=\beta \delta_{1, s_{i}}}=0
$$


where $\delta_{i, j}$ denotes the Kronecker delta. In conclusion, the nonzero part of the matrix representation of $f_{s_{i}}$ with respect to the above constructed basis of $\Psi_{s_{i}}$ is given by

$$
\operatorname{diag}\left(\frac{\epsilon_{i, 1}}{\left|u_{1}^{(i, 1)}\right|^{2}}, \ldots, \frac{\epsilon_{i, t_{i}}}{\left|u_{1}^{\left(i, t_{i}\right)}\right|^{2}}\right) .
$$

Thus, the signs of the perturbed pencil associated with $\hat{\lambda}$ at blocks of size $s_{i}$ are given by $\mathcal{L}_{s_{i}}^{\prime \prime}=\left\{\epsilon_{i, 1}, \ldots, \epsilon_{i, t_{i}}\right\}$ by Theorem 2.11, i.e., the signs associated with blocks of size $s_{i}$ are unchanged under perturbation in this case.

Blocks of type (ii): We consider blocks of size $1<s_{1}$ whenever $\beta \widehat{\lambda}=\alpha$, where we distinguish between the subcases of $s_{\nu}$ being equal to one or not.

First, let $s_{\nu}=1$. We construct a basis of the subspace $\Psi_{1}$ similar to (i). Since by Theorem 3.1, the pencil (3.4) generically has $t_{\nu}+1$ linearly independent Jordan chains of length 1 at $\hat{\lambda}$, clearly $x_{\nu, 1}, \ldots, x_{\nu, t_{\nu}}, e_{1}$ as in (3.6) can be chosen as the first vectors of a basis of $\Psi_{1}$. (We obtain a basis of $\Psi_{1}$ by adding a basis of $\Psi_{2}$ that is ignored here since $\operatorname{Ker}\left(G_{1}\right)=\Psi_{2}$.) Similarly to (i), we compute that $f_{1}\left(x_{\nu, k}, x_{\nu, \ell}\right)$ is the sum of on the one hand

$$
x_{\nu, k}^{*} F_{1} x_{\nu, \ell}=\underbrace{e_{1}^{*} F_{1} e_{1}}_{=0}-\underbrace{e_{1}^{*} F_{1} e_{\kappa_{\nu}+\ell}}_{=0}-\underbrace{e_{\kappa_{\nu}+k}^{*} F_{1} e_{1}}_{=0}+e_{\kappa_{\nu}+k}^{*} F_{1} e_{\kappa_{\nu}+\ell}= \begin{cases}\frac{\epsilon_{\nu, k}}{\left|u_{1}^{(\nu, k)}\right|^{2}} & \text { if } k=\ell, \\ 0 & \text { if } k \neq \ell,\end{cases}
$$

where we have made use of $1<s_{1}$, and on the other hand

$$
x_{\nu, k}^{*} F_{2} x_{\nu, \ell}=e_{1}^{*} F_{2} e_{1}-e_{1}^{*} F_{2} e_{\kappa_{\nu}+\ell}-e_{\kappa_{\nu}+k}^{*} F_{2} e_{1}+e_{\kappa_{\nu}+k}^{*} F_{2} e_{\kappa_{\nu}+\ell}=2 \beta-2 \beta=0
$$

for $k, \ell=1, \ldots, t_{\nu}$. Additionally, in this case we have

$$
f_{1}\left(x_{\nu, k}, e_{1}\right)=\underbrace{e_{1}^{*} F_{1} e_{1}}_{=0}-\underbrace{e_{\kappa_{\nu}+k}^{*} F_{1} e_{1}}_{=0}+\underbrace{e_{1}^{*} F_{2} e_{1}}_{=\beta}-\underbrace{e_{\kappa_{\nu}+k}^{*} F_{2} e_{1}}_{=\beta}=0
$$

and similarly $f_{1}\left(e_{1}, x_{\nu, \ell}\right)=0$ for $k, \ell=1, \ldots, t_{\nu}$. Finally, with $f_{1}\left(e_{1}, e_{1}\right)=\beta$ we obtain that the nonzero part of the matrix representation of $f_{1}$ with respect to the above constructed basis is given by

$$
\operatorname{diag}\left(\frac{\epsilon_{\nu, 1}}{\left|u_{1}^{(\nu, 1)}\right|^{2}}, \ldots, \frac{\epsilon_{\nu, t_{\nu}}}{\left|u_{1}^{\left(\nu, t_{\nu}\right)}\right|^{2}}, \beta\right) .
$$

From this, we read off $\mathcal{L}_{1}^{\prime \prime}=\left\{\epsilon_{\nu, 1}, \ldots, \epsilon_{\nu, t_{\nu}}, \operatorname{sgn}(\beta)\right\}$, i.e., the new block of size one that is created in this case (cf. Theorem 3.1) leads to the $\operatorname{sign} \operatorname{sgn}(\beta)$ being added to the list of signs at blocks of size one (recall that $\beta \neq 0$ is prescribed since $(\alpha, \beta) \neq 0$ ).

Now, the subcase $s_{\nu}>1$ is similar: Using Theorem 3.1, a basis of $\Psi_{1}$ can be constructed by from the vector $e_{1}$ by adding a basis of $\Psi_{2}$. We compute the nonzero part of the matrix representation to be given by $\left[e_{1}^{*} F e_{1}\right]=[\beta]$; thus $\mathcal{L}_{1}^{\prime \prime}=\{\operatorname{sgn}(\beta)\}$, i.e., the $\operatorname{sign} \operatorname{sgn}(\beta)$ will be attached to the new block of size one.

Blocks of type (iii): Generically, by Theorem 3.1 the pencil (3.4) has $t_{1}-1$ linearly independent Jordan chains of length $s_{1}$ at $\widehat{\lambda}$ and thus $\left\{x_{1,2}, \ldots, x_{1, t_{1}}\right\}$ is a basis of $\Psi_{s_{1}}$ 
(recall that $\Psi_{s_{1}+1}=\{0\}$ ). To compute the matrix representation of $f_{s_{1}}$ with respect to this basis, consider for $k, \ell=2, \ldots, t_{1}$ that $f_{s_{1}}\left(x_{1, k}, x_{1, \ell}\right)$ is the sum of on the one hand $x_{1, k}^{*} F_{1} x_{1, \ell}^{\left(s_{1}\right)}$ given by

$$
e_{1}^{*} F_{1} e_{s_{1}}-\underbrace{e_{1}^{*} F_{1} e_{\ell s_{1}}}_{=0}-\underbrace{e_{(k-1) s_{1}+1}^{*} F_{1} e_{s_{1}}}_{=0}+e_{(k-1) s_{1}+1}^{*} F_{1} e_{\ell s_{1}}= \begin{cases}\frac{\epsilon_{1, k}}{\left|u_{1}^{(1, k)}\right|^{2}}+\frac{\epsilon_{1,1}}{\left|u_{1}^{(1,1)}\right|^{2}} & \text { if } k=\ell, \\ \frac{\epsilon_{1,1}}{\left|u_{1}^{(1,1)}\right|^{2}} & \text { if } k \neq \ell,\end{cases}
$$

and on the other hand $x_{1, k}^{*} F_{2} x_{1, \ell}^{\left(s_{1}\right)}$ equal to

$$
e_{1}^{*} F_{2} e_{s_{1}}-e_{1}^{*} F_{2} e_{\ell_{1}}-e_{(k-1) s_{1}+1}^{*} F_{2} e_{s_{1}}+e_{(k-1) s_{1}+1}^{*} F_{2} e_{\ell_{1}}=2 \beta \delta_{1, s_{1}}-2 \beta \delta_{1, s_{1}}=0 .
$$

Therefore, the matrix representation of $f_{s_{1}}$ with respect to the above basis is given by

$$
M_{s_{1}}=\operatorname{diag}\left(\frac{\epsilon_{1,2}}{\left|u_{1}^{(1,2)}\right|^{2}}, \ldots, \frac{\epsilon_{1, t_{1}}}{\left|u_{1}^{\left(1, t_{1}\right)}\right|^{2}}\right)+\frac{\epsilon_{1,1}}{\left|u_{1}^{(1,1)}\right|^{2}}\left[\begin{array}{ccc}
1 & \ldots & 1 \\
\vdots & \ddots & \vdots \\
1 & \ldots & 1
\end{array}\right] \text {. }
$$

Clearly, the first term in $M_{s_{1}}$ (i.e., the diagonal matrix) is invertible. Also, it is a generic condition with respect to the entries of $u$ to assume that also $M_{s_{1}}$ itself is invertible. Therefore, by Lemma 2.12 the signs of the eigenvalues of $M_{s_{1}}$, that are equal to the list of signs of the perturbed pencil at blocks of size $s_{1}$ (denoted by $\mathcal{L}_{s_{1}}^{\prime \prime}$ ) is generically given by removing either exactly one sign -1 or one sign +1 from the list $\mathcal{L}_{s_{1}}=\left\{\epsilon_{1,1}, \ldots, \epsilon_{1, t_{1}}\right\}$.

Blocks of type (iv): Generically, by Theorem 3.1 the pencil (3.4) has $t_{1}$ linearly independent eigenvectors at $\hat{\lambda}$ and therefore $\left\{x_{1,2}, \ldots, x_{1, t_{1}}, e_{1}\right\}$ is a basis of $\Psi_{1}$ (recall that $\Psi_{2}=\{0\}$ in this case). We aim to compute the matrix representation of $f_{1}$ with respect to this basis as before. For $k, \ell=2, \ldots, t_{1}$, clearly $f_{1}\left(x_{1, k}, x_{1, \ell}\right)$ is the sum of on the one hand

$$
x_{1, k}^{*} F_{1} x_{1, \ell}=e_{1}^{*} F_{1} e_{1}-\underbrace{e_{1}^{*} F_{1} e_{\ell}}_{=0}-\underbrace{e_{k}^{*} F_{1} e_{1}}_{=0}+e_{k}^{*} F_{1} e_{\ell}= \begin{cases}\frac{\epsilon_{1, k}}{\left|u_{1}^{(1, k)}\right|^{2}}+\frac{\epsilon_{1,1}}{\left|u_{1}^{(1,1)}\right|^{2}} & \text { if } k=\ell, \\ \frac{\epsilon_{1,1}}{\left|u_{1}^{(1,1)}\right|^{2}} & \text { if } k \neq \ell,\end{cases}
$$

and on the other hand

$$
x_{1, k}^{*} F_{2} x_{1, \ell}=e_{1}^{*} F_{2} e_{1}-e_{1}^{*} F_{2} e_{\ell}-e_{k}^{*} F_{2} e_{1}+e_{k}^{*} F_{2} e_{\ell}=2 \beta-2 \beta=0 .
$$

Additionally, we need to compute

$$
f_{1}\left(x_{1, k}, e_{1}\right)=e_{1}^{*} F_{1} e_{1}-\underbrace{e_{k}^{*} F_{1} e_{1}}_{=0}+\underbrace{e_{1}^{*} F_{2} e_{1}}_{=\beta}-\underbrace{e_{k}^{*} F_{2} e_{1}}_{=\beta}=\frac{\epsilon_{1,1}}{\left|u_{1}^{(1,1)}\right|^{2}}
$$

and similarly $f_{1}\left(e_{1}, x_{1, \ell}\right)=\epsilon_{1,1} /\left|u_{1}^{(1,1)}\right|^{2}$ for $k, \ell=2, \ldots, t_{1}$. Finally, we have

$$
f_{1}\left(e_{1}, e_{1}\right)=e_{1}^{*} F_{1} e_{1}+e_{1}^{*} F_{2} e_{1}=\epsilon_{1,1} /\left|u_{1}^{(1,1)}\right|^{2}+\beta
$$


and therefore the matrix representation of $f_{1}$ with respect to the above basis is given by

$$
M_{1}=\operatorname{diag}\left(\frac{\epsilon_{1,2}}{\left|u_{1}^{(1,2)}\right|^{2}}, \ldots, \frac{\epsilon_{1, t_{1}}}{\left|u_{1}^{\left(1, t_{1}\right)}\right|^{2}}, \beta\right)+\frac{\epsilon_{1,1}}{\left|u_{1}^{(1,1)}\right|^{2}}\left[\begin{array}{ccc}
1 & \ldots & 1 \\
\vdots & \ddots & \vdots \\
1 & \ldots & 1
\end{array}\right] .
$$

Now, applying Lemma 2.12 as in the previous case (iii), the list of signs $\mathcal{L}_{1}^{\prime \prime}$ of (3.4) at blocks of size one is generically obtained by removing either exactly one sign -1 or exactly one sign +1 from the list $\mathcal{L}_{1}^{\prime}=\left\{\epsilon_{1,1}, \ldots, \epsilon_{1, t_{1}}, \operatorname{sgn}(\beta)\right\}$.

Remark 3.4 We note that there are results hidden in the statement and proof of Theorem 3.3 that are not at all obvious. First, consider blocks of type (ii): By Theorem 3.1 one such block is generically created under perturbation, and by Theorem 3.3 the sign consequently added to the list of signs $\mathcal{L}_{1}$ is generically $\operatorname{sgn}(\beta)$. But then, $\operatorname{sgn}(\beta)$ is exactly the sign that is attached to the eigenvalue $\widehat{\lambda}$ in the perturbation $(\beta \lambda-\alpha) u u^{*}$ in this case, i.e. the sign added due to one new block being created is generically the sign that is attached to $\widehat{\lambda}$ in the perturbation.

Then again, if blocks of type (iv) exist, by Theorem 3.1 the partial multiplicities of the perturbed pencil are generically unchanged, since both effects, one block being destroyed and a new block being created, neutralize one another. However, the list of signs $\mathcal{L}_{1}$ is generically unchanged under perturbation if no $\operatorname{sign} \operatorname{sgn}(-\beta)$ exists in $\mathcal{L}_{1}$, and otherwise, generically either one $\operatorname{sign} \operatorname{sgn}(-\beta)$ is replaced by $\operatorname{sgn}(\beta)$ or again $\mathcal{L}_{1}$ is unchanged. Consequently, the perturbed pencil is not generically prescribed to have the sign attached to $\widehat{\lambda}$ in the perturbation at one of its blocks in this case, which is again different from (ii).

To illustrate this remark, we consider the following example.

Example 3.5 The Hermitian $2 \times 2$ matrix pencil

$$
(E, A)=\left(\left[\begin{array}{ll}
1 & 0 \\
0 & 1
\end{array}\right],\left[\begin{array}{ll}
0 & 0 \\
0 & 0
\end{array}\right]\right) \in \mathbb{C}^{2,2} \times \mathbb{C}^{2,2}
$$

clearly consists of two Jordan blocks of size one with positive sign corresponding to 0 . We consider a Hermitian rank-1 perturbation of the form $\left(\beta u u^{*}, 0\right)$, where $u=\left[u_{1}, u_{2}\right]^{T} \in \mathbb{C}^{2}$ and $\beta \in \mathbb{R} \backslash\{0\}$. By Theorem 3.1 the perturbed pencil $\left(E+\beta u u^{*}, A\right)$ is generically regular and has two linearly independent Jordan chains of length one at 0 .

Clearly, a basis of the eigenspace of $\left(E+\beta u u^{*}, A\right)$ at 0 is given by $\left\{e_{1}, e_{2}\right\}$ and the matrix representation of the map $f_{1}$ with respect to that basis is given by

$$
M_{1}=\left[\begin{array}{cc}
1+\beta\left|u_{1}\right|^{2} & \beta \overline{u_{1}} u_{2} \\
\beta u_{1} \overline{u_{2}} & 1+\beta\left|u_{2}\right|^{2}
\end{array}\right] .
$$

Now, an elementary computation reveals that the eigenvalues of $M_{1}$ are given by 1 and $1+\beta\left(\left|u_{1}\right|^{2}+\left|u_{2}\right|^{2}\right)$. Thus, if either $\beta \geq 0$ or $\beta<0$ and $-1 / \beta>\left|u_{1}\right|^{2}+\left|u_{2}\right|^{2}$, the signs of the perturbed pencil at 0 are given by $\{+1,+1\}$ and if $\beta<0$ and $-1 / \beta<\left|u_{1}\right|^{2}+\left|u_{2}\right|^{2}$, these signs are given by $\{-1,+1\}$, which is in line with Theorem 3.3 and the above remark. 


\section{Hermitian rank-2 perturbations}

Before analyzing Hermitian rank-2 perturbations, we consider a further preliminary result. The following lemma deals with a class of matrices that includes the transformation matrix $S$ from Theorem 2.6.

Lemma 4.1 Let $n_{1} \geq \cdots \geq n_{m}>0$ be a series of integers and set $a:=n_{1}+\cdots+n_{m}$. Further, let $S$ have the shape

$$
S=\left[\begin{array}{cccc}
S_{1,1} & S_{1,2} & \cdots & S_{1, m} \\
& S_{2,2} & \ddots & \vdots \\
& & \ddots & S_{m-1, m} \\
& & & S_{m, m}
\end{array}\right] \in \mathbb{C}^{a, a},
$$

whereby all $S_{i, j} \in \mathbb{C}^{n_{j}, n_{j}}$ are upper triangular Toeplitz matrices. Then, $S^{-1}$ also has the shape (4.1), i.e., it is upper triangular and its $(i, j)$ block is an upper triangular Toeplitz matrix of dimension $n_{i} \times n_{j}$ for all $i$ and $j$.

This lemma is proven by straightforward computation using the well-known fact that the product of two upper triangular Toeplitz matrices is again an upper triangular Toeplitz matrix and that the inverse of an invertible upper triangular Toeplitz matrix is again one itself [11, Chapter 3]; details are omitted here. We go on to prove our main theorems on Hermitian rank-2 perturbations as in (2.19), i.e., ones of the form

$$
(\Delta E, \Delta A)=\left[\begin{array}{ll}
u & v
\end{array}\right]\left(\left[\begin{array}{ll}
0 & 1 \\
1 & 0
\end{array}\right],\left[\begin{array}{cc}
0 & \mu \\
\bar{\mu} & 0
\end{array}\right]\right)\left[\begin{array}{l}
u^{*} \\
v^{*}
\end{array}\right] .
$$

Hereby, we only consider the case $\mu \in \mathbb{C} \backslash \mathbb{R}$ since in case $\mu \in \mathbb{R}$, then (4.2) were the sum of two subsequent Hermitian rank-1 perturbations as covered in the previous section. We will analyze the Jordan structure under perturbation in Theorem 4.2 and the sign characteristic in Theorem 4.3.

Theorem 4.2 Let $(E, A) \in \mathbb{C}^{n, n} \times \mathbb{C}^{n, n}$ be regular and Hermitian with the partial multiplicities $n_{1} \geq \cdots \geq n_{m}>0$ associated with some eigenvalue $\widehat{\lambda} \in \mathbb{C}$. Then, for each $\mu \in \mathbb{C} \backslash \mathbb{R}$ there exists a generic set $\Omega_{\mu}^{\prime} \subseteq \mathbb{C}^{n} \times \mathbb{C}^{n}$, such that for all $(u, v) \in \Omega_{\mu}^{\prime},(E+\Delta E, A+\Delta A)$ as in (4.2) is regular and has the partial multiplicities $\left(n_{3}, \ldots, n_{m}\right)$ if $\widehat{\lambda} \notin\{\mu, \bar{\mu}\}$ and $\left(n_{3}, \ldots, n_{m}, 1\right)$ otherwise at $\widehat{\lambda}$.

Proof. We proceed similar to the proof of Theorem 3.1. Since by Theorem 2.6 and (2.16) the partial multiplicities of the perturbed pencil are generically greater than or equal to the ones given above, it again suffices to present one particular perturbation in each case that creates these partial multiplicities by Lemma 2.9. Let $(E, A)$ be in Hermitian Kronecker form as in Theorem 2.10 with the blocks at $\widehat{\lambda}$ coming first and ordered by their size. 
Case $\widehat{\lambda} \in \mathbb{C} \backslash \mathbb{R}$. Consider the first two blocks of $(E, A)$ associated with $\hat{\lambda}$ (each of which is paired to a block of the same size corresponding to the complex conjugate eigenvalue) and set $u:=e_{1}+e_{2 n_{1}+n_{2}+1}$ and $v:=e_{n_{1}+1}+e_{2 n_{1}+1}$. Then the first part of the perturbed pencil is given by

$$
\left[\begin{array}{ccc}
0 & -R_{n_{1}} J_{n_{1}}(\widehat{\lambda}-\lambda) \\
-R_{n_{1}} J_{n_{1}}(\overline{\widehat{\lambda}}-\lambda) & 0
\end{array}\right] \oplus\left[\begin{array}{cc}
0 & -R_{n_{2}} J_{n_{2}}(\widehat{\lambda}-\lambda) \\
-R_{n_{2}} J_{n_{2}}(\bar{\lambda}-\lambda) & 0
\end{array}\right]+(\lambda-\mu) u v^{*}+(\lambda-\bar{\mu}) v u^{*} .
$$

This matrix pencil can by permutations and multiplications with -1 of its rows be transformed to a matrix pencil of the type from the appendix of this paper. In fact, setting $\mu_{+}:=\bar{\lambda}-\lambda, \mu_{-}:=\lambda-\widehat{\lambda}, \nu_{+}:=\bar{\mu}-\lambda$, and $\nu_{-}:=\lambda-\mu$ in the matrix $T$ from the appendix, its determinant is computed to be equal to

$$
\begin{aligned}
& {\left[(\overline{\widehat{\lambda}}-\lambda)^{n_{1}}(\lambda-\widehat{\lambda})^{n_{2}}-(\overline{\widehat{\lambda}}-\lambda)^{n_{1}}(\bar{\mu}-\lambda)-(-1)^{n_{1}}(\lambda-\widehat{\lambda})^{n_{2}}(\bar{\mu}-\lambda)\right]} \\
& \cdot\left[(\lambda-\widehat{\lambda})^{n_{1}}(\overline{\widehat{\lambda}}-\lambda)^{n_{2}}+(-1)^{n_{2}}(\lambda-\widehat{\lambda})^{n_{1}}(\lambda-\mu)+(\bar{\lambda}-\lambda)^{n_{2}}(\lambda-\mu)\right] .
\end{aligned}
$$

This shows that in the above given blocks of the perturbed pencil, the eigenvalue $\hat{\lambda}$ does not occur if $\widehat{\lambda} \notin\{\mu, \bar{\mu}\}$ and occurs with algebraic multiplicity one otherwise. Since no other blocks of the perturbed pencil than these are perturbed, this particular perturbation clearly creates the desired partial multiplicities at $\hat{\lambda}$.

Case $\widehat{\lambda} \in \mathbb{R}$. Consider the first blocks of $(E, A)$ associated with $\widehat{\lambda}$ of sizes $n_{1}, n_{2}$ with signs $\epsilon_{1}, \epsilon_{2} \in\{ \pm 1\}$ and set $u:=e_{1}$ and $v:=e_{n_{1}+1}$. Then, the first blocks of the perturbed pencil are given by

$$
-\left(\epsilon_{1} R_{n_{1}} J_{n_{1}}(\widehat{\lambda}-\lambda) \oplus \epsilon_{2} R_{n_{2}} J_{n_{2}}(\widehat{\lambda}-\lambda)\right)+(\lambda-\mu) e_{1} e_{n_{1}+1}^{T}+(\lambda-\bar{\mu}) e_{n_{1}+1} e_{1}^{T}
$$

clearly not having the eigenvalue $\widehat{\lambda}$. Again, as no other blocks are perturbed, this perturbation creates the partial multiplicities $\left(n_{3}, \ldots, n_{m}\right)$ at $\hat{\lambda}$.

In the following theorem concerning the sign characteristic of Hermitian matrix pencils under rank-2 perturbations, we consider $(E, A)$ to have Jordan blocks of the sizes (3.2) at $\hat{\lambda}$. Hereby, we will employ both notations, i.e., the $n_{j}$ 's and the $s_{i}$ 's depending on which is more convenient. Finally, let $\Omega_{\mu}^{\prime}$ denote the generic set from Theorem 4.2.

Theorem 4.3 Let $(E, A) \in \mathbb{C}^{n, n} \times \mathbb{C}^{n, n}$ be regular and Hermitian and at some eigenvalue $\widehat{\lambda} \in \mathbb{R}$, let $(E, A)$ have the partial multiplicities (3.2) and the list of signs $\mathcal{L}_{s_{i}}$ attached to blocks of size $s_{i}$ for $i=1, \ldots, \nu$. Then, for each $\mu \in \mathbb{C} \backslash \mathbb{R}$ there exists a generic set $\Omega_{\mu} \subseteq \Omega_{\mu}^{\prime}$, such that for all $(u, v) \in \Omega_{\mu}$ the list of signs $\mathcal{L}_{s_{i}}^{\prime \prime}$ at blocks of size $s_{i}$ of $(E+\Delta E, A+\Delta A)$ as in (4.2) is obtained by subsequently executing the following steps:

- If $i=1$, then $\mathcal{L}_{s_{i}}^{\prime}$ is obtained from $\mathcal{L}_{s_{i}}$ by removing either exactly one sign -1 or exactly one sign +1 , else $\mathcal{L}_{s_{i}}^{\prime}:=\mathcal{L}_{s_{i}}$. 
- If either $i=1$ and $t_{1} \geq 2$ or $i=2$ and $t_{1}=1$, then $\mathcal{L}_{s_{i}}^{\prime \prime}$ is obtained from $\mathcal{L}_{s_{i}}^{\prime}$ by removing exactly one sign -1 or exactly one sign +1 , else $\mathcal{L}_{s_{i}}^{\prime \prime}:=\mathcal{L}_{s_{i}}^{\prime}$.

Proof. We proceed similar to the proof of Theorem 3.3. Assuming $(E, A)$ to be in Hermitian Kronecker form as in Theorem 2.10 with the $\widehat{\lambda}$ blocks coming first, these first blocks of $E(E, A)$ are in Weierstraß canonical form. Now, let the generic condition from Theorem 2.6 on $u, v$ be satisfied, so that there is an invertible matrix $S \in \mathbb{C}^{n, n}$ as in (2.10) for which we have

$$
\left[\begin{array}{l}
u^{*} \\
v^{*}
\end{array}\right] S^{-1}=\left[\begin{array}{cccccccc}
e_{1, n_{1}}^{T} & 0 & e_{1, n_{3}}^{T} & 0 & e_{1, n_{5}}^{T} & \ldots & \sigma e_{1, n_{m}}^{T} & M \\
x^{T} & e_{1, n_{2}}^{T} & 0 & e_{1, n_{4}}^{T} & 0 & & \rho e_{1, n_{m}}^{T} &
\end{array}\right]
$$

for certain $x=\left[x_{1}, \ldots, x_{n_{1}}\right]^{T} \in \mathbb{C}^{n_{1}}$ and $M$ of suitable size. Thus, $S E(E+\Delta E, A+\Delta A) S^{-1}$ is in rank-2 partial Brunovsky form as in Theorem 2.6 and thus has the Jordan chains (2.7)(2.9) at $\widehat{\lambda}$. But also

$$
S^{-*}(E+\Delta E, A+\Delta A) S^{-1}
$$

has these chains at $\hat{\lambda}$ and in addition, it has the same sign characteristic as $(E+\Delta E, A+$ $\Delta A)$. By Lemma 4.1, the matrix $S^{-1}$ has the structure

$$
S^{-1}=\left[\begin{array}{cccc}
S_{1,1} & S_{1,2} & \cdots & S_{1, m} \\
& S_{2,2} & \ddots & \vdots \\
& & \ddots & S_{m-1, m} \\
& & & S_{m, m}
\end{array}\right] \oplus I_{n-a} \in \mathbb{C}^{n, n},
$$

whereby $a=n_{1}+\cdots+n_{m}$ and each $S_{i, j}$ has dimension $n_{i} \times n_{j}$ but is still an upper triangular Toeplitz matrix, i.e., if $n_{i}>n_{j}$ then $S_{i, j}$ has $n_{i}-n_{j}$ all-zero rows at the bottom. Also, let us denote the $(1,1)$-entry of each $S_{i, j}$ by $s_{i, j}$ with $s_{i, i} \neq 0$ for $i=1, \ldots, m$. Now, the sign characteristic of (4.4) can be extracted from the following matrix

$$
F:=F_{1}+F_{2}:=S^{-*} E S^{-1}+S^{-*}\left[\begin{array}{ll}
u & v
\end{array}\right] R_{2}\left[\begin{array}{c}
u^{*} \\
v^{*}
\end{array}\right] S^{-1} .
$$

In the remainder of this proof, we denote by $\epsilon_{1}, \ldots, \epsilon_{m}$ the signs of $(E, A)$ attached to its blocks at $\hat{\lambda}$, so that $\epsilon_{j}$ is the sign of the $j$ th diagonal block of $(E, A)$. Then, the topleft $a \times a$ block of $F_{1}$ is given by

$$
\left[\begin{array}{ccccc}
\epsilon_{1} S_{1,1}^{*} R_{n_{1}} S_{1,1} & \epsilon_{1} S_{1,1}^{*} R_{n_{1}} S_{1,2} & \epsilon_{1} S_{1,1}^{*} R_{n_{1}} S_{1,3} & \ldots & \epsilon_{1} S_{1,1}^{*} R_{n_{1}} S_{1, m} \\
\epsilon_{1} S_{1,2}^{*} R_{n_{1}} S_{1,1} & \sum_{j=1}^{2} \epsilon_{j} S_{j, 2}^{*} R_{n_{j}} S_{j, 2} & \sum_{j=1}^{2} \epsilon_{j} S_{j, 2}^{*} R_{n_{j}} S_{j, 3} & \ldots & \sum_{j=1}^{2} \epsilon_{j} S_{j, 2}^{*} R_{n_{j}} S_{j, m} \\
\epsilon_{1} S_{1,3}^{*} R_{n_{1}} S_{1,1} & \sum_{j=1}^{2} \epsilon_{j} S_{j, 3}^{*} R_{n_{j}} S_{j, 2} & \sum_{j=1}^{3} \epsilon_{j} S_{j, 3}^{*} R_{n_{j}} S_{j, 3} & \ddots & \\
\vdots & \vdots & \ddots & \ddots & \vdots \\
\epsilon_{1} S_{1, m}^{*} R_{n_{1}} S_{1,1} & \sum_{j=1}^{2} \epsilon_{j} S_{j, m}^{*} R_{n_{j}} S_{j, 2} & & \ldots & \sum_{j=1}^{m} \epsilon_{j} S_{j, m}^{*} R_{n_{j}} S_{j, m}
\end{array}\right]
$$


We note that for $1 \leq j \leq \min (k, \ell)$, the $j$ th summand of the $(k, \ell)$ block of the above matrix is given by

$$
\epsilon_{j} S_{j, k}^{*} R_{n_{j}} S_{j, \ell}=\epsilon_{j}\left[\begin{array}{cc}
0 & 0 \\
0 & R_{d} \operatorname{Toep}(w)
\end{array}\right] \in \mathbb{C}^{n_{k}, n_{\ell}}
$$

for some $w \in \mathbb{C}^{d}$ setting $d:=n_{k}+n_{\ell}-n_{j}$. In particular, its $\left(1, n_{\ell}\right)$ entry is zero whenever $n_{\ell}<n_{j}$ and its $\left(n_{k}, 1\right)$ entry is zero whenever $n_{k}<n_{j}$. Also, by (4.3) it is clear that the topleft $a \times a$ block of $F_{2}$ is equal to

$$
\left[\begin{array}{cccccc}
e_{1, n_{1}} x^{*}+x e_{1, n_{1}}^{T} & e_{1, n_{1}} e_{1, n_{2}}^{T} & x e_{1, n_{3}}^{T} & e_{1, n_{1}} e_{1, n_{4}}^{T} & x e_{1, n_{5}}^{T} & \ddots \\
e_{1, n_{2}} e_{1, n_{1}}^{T} & 0 & e_{1, n_{2}} e_{1, n_{3}}^{T} & 0 & e_{1, n_{2}} e_{1, n_{5}}^{T} & \ddots \\
e_{1, n_{3}} x^{*} & e_{1, n_{3}} e_{1, n_{2}}^{T} & 0 & e_{1, n_{3}} e_{1, n_{4}}^{T} & \ddots & \\
e_{1, n_{4}} e_{1, n_{1}}^{T} & 0 & e_{1, n_{4}} e_{1, n_{3}}^{T} & \ddots & & \vdots \\
e_{1, n_{5}} x^{*} & e_{1, n_{5}} e_{1, n_{2}}^{T} & \ddots & & 0 & e_{1, n_{m-1}} e_{1, n_{m}}^{T} \\
\ddots & \ddots & & \ldots & e_{1, n_{m}} e_{1, n_{m-1}}^{T} & 0
\end{array}\right],
$$

and that these topleft $a \times a$ blocks of $F_{1}$ and $F_{2}$ are conformably partitioned, i.e., the $(k, \ell)$ block of each matrix has dimension $n_{k} \times n_{\ell}$.

Now, by Theorem 2.11 for each $i=1, \ldots, \nu$ the signs of (4.4) at blocks of size $s_{i}$ are equal to the signs in the inertia of the selfadjoint map $G_{s_{i}}: \Psi_{s_{i}} \rightarrow \Psi_{s_{i}}$, where we have

$$
\begin{aligned}
\Psi_{s_{i}}=\left\{x \in \mathbb{C}^{n} \backslash\{0\} \mid\right. & \hat{\lambda}(E+\Delta E) x=(A+\Delta A) x \text { and } x \text { can be extended to } \\
& \text { a chain of } \left.(E+\Delta E, A+\Delta A) \text { of at least length } s_{i}\right\} \cup\{0\} .
\end{aligned}
$$

This inertia shall be extracted by computing a matrix representation of

$$
f_{s_{i}}: \Psi_{s_{i}} \times \Psi_{s_{i}} \rightarrow \mathbb{C}, \quad(x, y) \mapsto x^{*} G_{s_{i}} y=x^{*} F y^{\left(s_{i}\right)}
$$

with respect to a suitable basis of $\Psi_{s_{i}}$. By Theorem 4.2, there generically exist blocks of the following sizes in (4.4) at $\widehat{\lambda}$ :

(i) blocks of size $s_{i}$ with $s_{i}<n_{3}$,

(ii) blocks of size $n_{3}$.

Blocks of type (i): We consider the Jordan chains of length $s_{i}$ of (4.4) from (2.8)-(2.9). Letting $\kappa_{i}=s_{1} t_{1}+\cdots+s_{i-1} t_{i-1}$ as before and also $\eta_{i}:=t_{1}+\cdots+t_{i-1}$, we introduce the following notation for these chains:

$$
y_{i, k}^{(j)}:= \begin{cases}e_{n_{1}+j}-e_{\kappa_{i}+(k-1) s_{i}+j}, & \text { if } k \text { and } \eta_{i} \text { are both odd or both even } \\ e_{n_{1}+n_{2}+j}-e_{\kappa_{i}+(k-1) s_{i}+j}, & \text { otherwise }\end{cases}
$$


for $j=1, \ldots, s_{i}$ and $k=1, \ldots, t_{i}$.

As in the previous section, we aim to extract the signs of a matrix representation of $f_{s_{i}}$ by considering a basis of $\Psi_{s_{i}}$, whose last $\operatorname{dim}\left(\Psi_{s_{i}+1}\right)$ vectors form a basis of $\Psi_{s_{i}+1}$. Since by Theorem 4.2 the pencil (4.4) generically has $t_{i}$ linearly independent Jordan chains of length $s_{i}$ at $\hat{\lambda}$, the first $t_{i}=\operatorname{dim}\left(\Psi_{s_{i}+1}\right)-\operatorname{dim}\left(\Psi_{s_{i}}\right)$ vectors of this basis can be chosen as $y_{i, 1}, \ldots, y_{i, t_{i}}$ as in (4.7) (omitting the superscript if equal to one). But as basis vectors in $\Psi_{s_{i}+1}$ lie in $\operatorname{Ker} G_{s_{i}}$, we ignore them when computing a matrix representation of $f_{s_{i}}$.

For simplicity, we assume in the following that $\eta_{i}$ is odd but the other case is entirely analogous. In order to compute $f_{s_{i}}\left(y_{i, k}, y_{i, \ell}\right)$ for all $k, \ell \in\left\{1, \ldots, t_{i}\right\}$, we may consider the terms $y_{i, k}^{*} F_{1} y_{i, \ell}^{\left(s_{i}\right)}$ and $y_{i, k}^{*} F_{2} y_{i, \ell}^{\left(s_{i}\right)}$ separately because of (4.5). Recalling that the signs of $(E, A)$ associated with blocks of size $s_{i}$ are given by $\mathcal{L}_{s_{i}}=\left\{\epsilon_{\eta_{i}+1}, \epsilon_{\eta_{i}+2}, \ldots, \epsilon_{\eta_{i}+t_{i}}\right\}$, we compute in the following subcases:

Subcase $k$ is odd and $\ell$ is even: We have that $y_{i, k}^{*} F_{1} y_{i, \ell}^{\left(s_{i}\right)}$ is given by

$$
\begin{array}{r}
\underbrace{e_{n_{1}+1}^{T} F_{1} e_{n_{1}+n_{2}+s_{i}}}_{=0}-\underbrace{e_{n_{1}+1}^{T} F_{1} e_{\kappa_{i}+\ell s_{i}}}_{=0}-\underbrace{e_{\kappa_{i}+(k-1) s_{i}+1}^{T} F_{1} e_{n_{1}+n_{2}+s_{i}}}_{=0}+e_{\eta_{i}+\min (k, \ell)}^{T} e_{\kappa_{i}+(k-1) s_{i}+1} F_{1} e_{\kappa_{i}+\ell s_{i}} \\
=\sum_{j=\eta_{i}+1} \epsilon_{j} \overline{s_{j, \eta_{i}+k}} s_{j, \eta_{i}+\ell} .
\end{array}
$$

Hereby, the first three terms were simplified using $n_{3}>s_{i}$ and the last equality was obtained by regarding the $\left(1, s_{i}\right)$-entry of the $\left(\eta_{i}+k, \eta_{i}+\ell\right)$-block of $F_{1}$, which is the sum of matrices of the form (4.6); the lower summation bound arises as the desired $\left(1, s_{i}\right)$ entry is 0 whenever $s_{i}<n_{j}$ (whereby $j$ is the summation index). On the other hand, the term $y_{i, k}^{*} F_{2} y_{i, \ell}^{\left(s_{i}\right)}$ from above is equal to

$$
\underbrace{e_{n_{1}+1}^{T} F_{2} e_{n_{1}+n_{2}+s_{i}}}_{=\delta_{1, s_{i}}}-\underbrace{e_{n_{1}+1}^{T} F_{2} e_{\kappa_{i}+\ell s_{i}}}_{=\delta_{1, s_{i}}}-\underbrace{e_{\kappa_{i}+(k-1) s_{i}+1}^{T} F_{2} e_{n_{1}+n_{2}+s_{i}}}_{=\delta_{1, s_{i}}}+\underbrace{e_{\kappa_{i}+(k-1) s_{i}+1}^{T} F_{2} e_{\kappa_{i}+\ell s_{i}}}_{=\delta_{1, s_{i}}}=0,
$$

where $\delta_{i, j}$ is the Kronecker delta and we used that $\eta_{i}+k$ is even and $\eta_{i}+\ell$ is odd. Thus, in the case that $k$ is odd and $\ell$ is even, we obtain

$$
f_{s_{i}}\left(y_{i, k}, y_{i, \ell}\right)=\sum_{j=\eta_{i}+1}^{\eta_{i}+\min (k, \ell)} \epsilon_{j} \overline{s_{j, \eta_{i}+k}} s_{j, \eta_{i}+\ell}
$$

Then again, if $k$ is even and $\ell$ is odd, the same result is obtained since the map $f_{s_{i}}$ is Hermitian (recall that $G_{s_{i}}$ is self-adjoint). However, the remaining cases that $k$ and $\ell$ are both odd or both even are treated similarly:

Subcase $k$ and $\ell$ are both odd: We obtain that $y_{i, k}^{*} F_{1} y_{i, \ell}^{\left(s_{i}\right)}$ is equal to

$$
\begin{array}{r}
\underbrace{e_{n_{1}+1}^{T} F_{1} e_{n_{1}+s_{i}}}_{=0}-\underbrace{e_{n_{1}+1}^{T} F_{1} e_{\kappa_{i}+\ell s_{i}}}_{=0}-\underbrace{e_{\kappa_{i}+(k-1) s_{i}+1}^{T} F_{1} e_{n_{1}+s_{i}}}_{=0}+e_{\kappa_{i}+(k-1) s_{i}+1}^{T} F_{1} e_{\kappa_{i}+\ell s_{i}} \\
=\sum_{\eta_{i}+\min (k, \ell)} \epsilon_{j} \overline{s_{j, \eta_{i}+k}} s_{j, \eta_{i}+\ell},
\end{array}
$$


where the first three terms were simplified using $n_{3}>s_{i}$ and the last equality was obtained exactly as described in the above subcase. Then, the other term $y_{i, k}^{*} F_{2} y_{i, \ell}^{\left(s_{i}\right)}$ is equal to

$$
e_{n_{1}+1}^{T} F_{2} e_{n_{1}+s_{i}}-e_{n_{1}+1}^{T} F_{2} e_{\kappa_{i}+\ell s_{i}}-e_{\kappa_{i}+(k-1) s_{i}+1}^{T} F_{2} e_{n_{1}+s_{i}}+e_{\kappa_{i}+(k-1) s_{i}+1}^{T} F_{2} e_{\kappa_{i}+\ell s_{i}}=0,
$$

since every one of the four terms is already zero (recall that $\eta_{i}+k$ and $\eta_{i}+\ell$ are both even). Thus, the equation (4.8) is obtained in this subcase as well.

Subcase $k$ and $\ell$ are both even: Then, $y_{i, k}^{*} F_{1} y_{i, \ell}^{\left(s_{i}\right)}$ is computed to be

$$
\begin{array}{r}
\underbrace{e_{n_{1}+n_{2}+1}^{T} F_{1} e_{n_{1}+n_{2}+s_{i}}}_{=0}-\underbrace{e_{n_{1}+n_{2}+1}^{T} F_{1} e_{\kappa_{i}+\ell s_{i}}}_{=0}-\underbrace{e_{\kappa_{i}+(k-1) s_{i}+1}^{T} F_{1} e_{n_{1}+n_{2}+s_{i}}}_{=0}+\underbrace{T}_{\kappa_{i}+(k-1) s_{i}+1} F_{1} e_{\kappa_{i}+\ell s_{i}} \\
=\sum_{j=\eta_{i}+1}^{T+\min (k, \ell)} \epsilon_{j} \overline{s_{j, \eta_{i}+k}} s_{j, \eta_{i}+\ell}
\end{array}
$$

just as in the previous subcases. The remaining term $y_{i, k}^{*} F_{2} y_{i, \ell}^{\left(s_{i}\right)}$ is equal to

$e_{n_{1}+n_{2}+1}^{T} F_{2} e_{n_{1}+n_{2}+s_{i}}-e_{n_{1}+n_{2}+1}^{T} F_{2} e_{\kappa_{i}+\ell s_{i}}-e_{\kappa_{i}+(k-1) s_{i}+1}^{T} F_{2} e_{n_{1}+n_{2}+s_{i}}+e_{\kappa_{i}+(k-1) s_{i}+1}^{T} F_{2} e_{\kappa_{i}+\ell s_{i}}$,

which is equal to zero, since all of the four terms are already zero (recall that $\eta_{i}+k$ and $\eta_{i}+\ell$ are both odd). Hence, (4.8) also holds in this subcase.

Clearly, the nonzero part of the desired matrix representation of $f_{s_{i}}$ is given by $M_{s_{i}}=$ $\left[f_{s_{i}}\left(y_{i, k}, y_{i, \ell}\right)\right]_{k \ell}$. We apply a series of $*$-congruence transformations to $M_{s_{i}}$ : First, add the $-\left(\overline{s_{\eta_{i}+1, \eta_{i}+j}}\right) /\left(\overline{s_{\eta_{i}+1, \eta_{i}+1}}\right)$-multiple of the first row onto the $j$ th row and the $-\left(s_{\eta_{i}+1, \eta_{i}+j}\right) /$ $\left(s_{\eta_{i}+1, \eta_{i}+1}\right)$-multiple of the first column onto the $j$ th column for $j=1,2, \ldots, t_{i}$, then repeat with the second row/column, then with the third, and so on, which yields the matrix

$$
\operatorname{diag}\left(\epsilon_{\eta_{i}+1}\left|s_{\eta_{i}+1, \eta_{i}+1}\right|^{2}, \epsilon_{\eta_{i}+2}\left|s_{\eta_{i}+2, \eta_{i}+2}\right|^{2}, \ldots, \epsilon_{\eta_{i}+t_{i}}\left|s_{\eta_{i}+t_{i}, \eta_{i}+t_{i}}\right|^{2}\right) .
$$

Since the signs of the perturbed pencil at blocks of size $s_{i}$ are given by the signs of the eigenvalues of $M_{s_{i}}$, they are read off to be equal to $\mathcal{L}_{s_{i}}^{\prime \prime}=\left\{\epsilon_{\eta_{i}+1}, \epsilon_{\eta_{i}+2}, \ldots, \epsilon_{\eta_{i}+t_{i}}\right\}$, i.e., the original signs.

Blocks of type (ii): To extract the signs at blocks of size $n_{3}$, we employ a different set of chains of length $n_{3}$ than the ones from Theorem 2.6 given in (2.7)-(2.9). Letting $\tau$ be the number of linearly independent chains of $(E, A)$ at $\widehat{\lambda}$ with length at least $n_{3}$, one can verify as in the proof of Theorem 2.6 that for each $k=3,4, \ldots, \tau$ the vectors

$$
z_{k}^{(j)}:= \begin{cases}e_{j}-e_{n_{1}+\cdots+n_{k-1}+j}-\left[\begin{array}{lll}
e_{n_{1}+j} & \ldots & e_{n_{1}+1}
\end{array}\right]\left[\begin{array}{lll}
x_{1} \ldots & \ldots & x_{j}
\end{array}\right]^{T}, \quad j=1, \ldots, n_{3}, \quad \text { if } k \text { is odd } \\
e_{n_{1}+j}-e_{n_{1}+\cdots+n_{k-1}+j}, & \text { is even }\end{cases}
$$

form a Jordan chain of (4.4) with length $n_{3}$. Since by Theorem 4.2 there generically exist $\tau-2$ linearly independent Jordan chains of length $n_{3}$ of (4.4) at $\hat{\lambda}$, the set $\left\{z_{3}, \ldots, z_{\tau}\right\}$ is a basis of $\Psi_{n_{3}}$ (recall that $\left.\Psi_{n_{3}+1}=\{0\}\right)$. Thus, in the following we compute $f_{n_{3}}\left(z_{k}, z_{\ell}\right)=$ $z_{k}^{*} F_{1} z_{\ell}^{\left(n_{3}\right)}+z_{k}^{*} F_{2} z_{\ell}^{\left(n_{3}\right)}$ for $k, \ell \in\{3, \ldots, \tau\}$ to obtain the signs of the perturbed pencil (4.4). 
First, let us compute the term $z_{k}^{*} F_{2} z_{\ell}^{\left(n_{3}\right)}$, whereby we again consider the three subcases depending on $k$ and $\ell$ being odd or even. Setting $\omega_{k}:=n_{1}+\cdots+n_{k-1}$, in the first subcase we assume that $k$ and $\ell$ are both odd. Then, $z_{k}^{*} F_{2} z_{\ell}^{\left(n_{3}\right)}$ is given by

$$
\begin{aligned}
& \left(e_{1}^{T}-e_{\omega_{k}+1}^{T}-\overline{x_{1}} e_{n_{1}+1}^{T}\right) F_{2}\left(e_{n_{3}}-e_{\omega_{\ell}+n_{3}}-x_{1} e_{n_{1}+n_{3}}-\cdots-x_{n_{3}} e_{n_{1}+1}\right) \\
= & e_{1}^{T} F_{2} e_{n_{3}}-e_{1}^{T} F_{2} e_{\omega_{\ell}+n_{3}}-x_{1} e_{1}^{T} F_{2} e_{n_{1}+n_{3}}-\cdots-x_{n_{3}} e_{1}^{T} F_{2} e_{n_{1}+1} \\
& -e_{\omega_{k}+1}^{T} F_{2} e_{n_{3}}+e_{\omega_{k}+1}^{T} F_{2} e_{\omega_{\ell}+n_{3}}+x_{1} e_{\omega_{k}+1}^{T} F_{2} e_{n_{1}+n_{3}}+\cdots+x_{n_{3}} e_{\omega_{k}+1}^{T} F_{2} e_{n_{1}+1} \\
& -\overline{x_{1}} e_{n_{1}+1}^{T} F_{2} e_{n_{3}}+\overline{x_{1}} e_{n_{1}+1}^{T} F_{2} e_{\omega_{\ell}+n_{3}}+\left|x_{1}\right|^{2} e_{n_{1}+1}^{T} F_{2} e_{n_{1}+n_{3}}+\cdots+\overline{x_{1}} x_{n_{3}} e_{n_{1}+1}^{T} F_{2} e_{n_{1}+1} .
\end{aligned}
$$

The nonzero terms occurring in this computation are the ones:

$$
\begin{array}{ccc}
e_{1}^{T} F_{2} e_{n_{3}}=\overline{x_{n_{3}}}+x_{1} \delta_{1, n_{3}}, & -e_{1}^{T} F_{2} e_{\omega_{\ell}+n_{3}}=-x_{1} \delta_{1, n_{3}}, & -x_{n_{3}} e_{1}^{T} F_{2} e_{n_{1}+1}=-x_{n_{3}}, \\
-e_{\omega_{k}+1}^{T} F_{2} e_{n_{3}}=-\overline{x_{n_{3}}}, & x_{n_{3}} e_{\omega_{k}+1}^{T} F_{2} e_{n_{1}+1}=x_{n_{3}}, & -\overline{x_{1}} e_{n_{1}+1}^{T} F_{2} e_{n_{3}}=-\overline{x_{1}} \delta_{1, n_{3}}, \\
\overline{x_{1}} e_{n_{1}+1}^{T} F_{2} e_{\omega_{\ell}+n_{3}}=\overline{x_{1}} \delta_{1, n_{3}}, &
\end{array}
$$

so that clearly, we obtain $z_{k}^{*} F_{2} z_{\ell}^{\left(n_{3}\right)}=0$ in this subcase (recall that $\delta_{i, j}$ is the Kronecker delta). Similarly, if $k$ is odd and $\ell$ is even, $z_{k}^{*} F_{2} z_{\ell}^{\left(n_{3}\right)}$ is given by

$$
\begin{aligned}
& \left(e_{1}^{T}-e_{\omega_{k}+1}^{T}-\overline{x_{1}} e_{n_{1}+1}^{T}\right) F_{2}\left(e_{n_{1}+n_{3}}-e_{\omega_{\ell}+n_{3}}\right)=e_{1}^{T} F_{2} e_{n_{1}+n_{3}}-e_{1}^{T} F_{2} e_{\omega_{\ell}+n_{3}} \\
& -e_{\omega_{k}+1}^{T} F_{2} e_{n_{1}+n_{3}}+e_{\omega_{k}+1}^{T} F_{2} e_{\omega_{\ell}+n_{3}}-\overline{x_{1}} e_{n_{1}+1}^{T} F_{2} e_{n_{1}+n_{3}}+\overline{x_{1}} e_{n_{1}+1}^{T} F_{2} e_{\omega_{\ell}+n_{3}}=0 .
\end{aligned}
$$

Hereby, the result $z_{k}^{*} F_{2} z_{\ell}^{\left(n_{3}\right)}=0$ is obtained since the last two terms above are zero (recall that $\ell$ is even) and the first four terms are each equal to $\pm \delta_{1, n_{3}}$ so that they exactly cancel out. (We remind that the case that $k$ is even and $\ell$ is odd will later follow from this case as $f_{n_{3}}$ is Hermitian.) Finally, whenever $k$ and $\ell$ are both even, the term $z_{k}^{*} F_{2} z_{\ell}^{\left(n_{3}\right)}$ is equal to

$$
\begin{aligned}
& \left(e_{n_{1}+1}^{T}-e_{\omega_{k}+1}^{T}\right) F_{2}\left(e_{n_{1}+n_{3}}-e_{\omega_{\ell}+n_{3}}\right)=e_{n_{1}+1}^{T} F_{2} e_{n_{1}+n_{3}}-e_{n_{1}+1}^{T} F_{2} e_{\omega_{\ell}+n_{3}} \\
& -e_{\omega_{k}+1}^{T} F_{2} e_{n_{1}+n_{3}}+e_{\omega_{k}+1}^{T} F_{2} e_{\omega_{\ell}+n_{3}}=0,
\end{aligned}
$$

where the sum is zero since each of the four terms above is zero itself.

It remains to compute the other term $z_{k}^{*} F_{1} z_{\ell}^{\left(n_{3}\right)}$ for all $k, \ell \in\{3, \ldots, \tau\}$, but in addition to the subcases from above, we have to account for another thing. Since the results depend the list of signs of $(E, A)$ attached to blocks of size $n_{3}$, denoted by $\mathcal{L}_{n_{3}}$, we distinguish between the following cases:

case (a): $n_{2}>n_{3}$, since then $\mathcal{L}_{n_{3}}=\left\{\epsilon_{3}, \ldots, \epsilon_{\tau}\right\}$,

case (b): $n_{1}>n_{2}=n_{3}$, since then $\mathcal{L}_{n_{3}}=\left\{\epsilon_{2}, \ldots, \epsilon_{\tau}\right\}$,

case (c): $n_{1}=n_{2}=n_{3}$, since then $\mathcal{L}_{n_{3}}=\left\{\epsilon_{1}, \ldots, \epsilon_{\tau}\right\}$. 
Case (a): For odd $k$ and $\ell$ we obtain that $z_{k}^{*} F_{1} z_{\ell}^{\left(n_{3}\right)}$ is equal to

$$
\begin{aligned}
& \left(e_{1}^{T}-e_{\omega_{k}+1}^{T}-\overline{x_{1}} e_{n_{1}+1}^{T}\right) F_{1}\left(e_{n_{3}}-e_{\omega_{\ell}+n_{3}}-x_{1} e_{n_{1}+n_{3}}-\cdots-x_{n_{3}} e_{n_{1}+1}\right) \\
= & e_{1}^{T} F_{1} e_{n_{3}}-e_{1}^{T} F_{1} e_{\omega_{\ell}+n_{3}}-x_{1} e_{1}^{T} F_{1} e_{n_{1}+n_{3}}-\cdots-x_{n_{3}} e_{1}^{T} F_{1} e_{n_{1}+1} \\
& -e_{\omega_{k}+1}^{T} F_{1} e_{n_{3}}+e_{\omega_{k}+1}^{T} F_{1} e_{\omega_{\ell}+n_{3}}+x_{1} e_{\omega_{k}+1}^{T} F_{1} e_{n_{1}+n_{3}}+\cdots+x_{n_{3}} e_{\omega_{k}+1}^{T} F_{1} e_{n_{1}+1} \\
& -\overline{x_{1}} e_{n_{1}+1}^{T} F_{1} e_{n_{3}}+\overline{x_{1}} e_{n_{1}+1}^{T} F_{1} e_{\omega_{\ell}+n_{3}}+\left|x_{1}\right|^{2} e_{n_{1}+1}^{T} F_{1} e_{n_{1}+n_{3}}+\cdots+\overline{x_{1}} x_{n_{3}} e_{n_{1}+1}^{T} F_{1} e_{n_{1}+1} \\
= & \sum_{j=3}^{\min (k, \ell)} \epsilon_{j} \overline{s_{j, k}} s_{j, \ell},
\end{aligned}
$$

since all terms other than $e_{\omega_{k}+1}^{T} F_{1} e_{\omega_{\ell}+n_{3}}$ are equal to zero. On the other hand, if $k$ is odd and $\ell$ is even, $z_{k}^{*} F_{1} z_{\ell}^{\left(n_{3}\right)}$ is equal to

$$
\begin{aligned}
& \left(e_{1}^{T}-e_{\omega_{k}+1}^{T}-\overline{x_{1}} e_{n_{1}+1}^{T}\right) F_{1}\left(e_{n_{1}+n_{3}}-e_{\omega_{\ell}+n_{3}}\right)=e_{1}^{T} F_{1} e_{n_{1}+n_{3}}-e_{1}^{T} F_{1} e_{\omega_{\ell}+n_{3}} \\
& -e_{\omega_{k}+1}^{T} F_{1} e_{n_{1}+n_{3}}+e_{\omega_{k}+1}^{T} F_{1} e_{\omega_{\ell}+n_{3}}-\overline{x_{1}} e_{n_{1}+1}^{T} F_{1} e_{n_{1}+n_{3}}+\overline{x_{1}} e_{n_{1}+1}^{T} F_{1} e_{\omega_{\ell}+n_{3}}=\sum_{j=3}^{\min (k, \ell)} \epsilon_{j} \overline{s_{j, k}} s_{j, \ell}
\end{aligned}
$$

since again only the term $e_{\omega_{k}+1}^{T} F_{1} e_{\omega_{\ell}+n_{3}}$ contributes to the result. Finally, if $k$ and $\ell$ are both even, then $z_{k}^{*} F_{1} z_{\ell}^{\left(n_{3}\right)}$ is given by

$$
\begin{aligned}
& \left(e_{n_{1}+1}^{T}-e_{\omega_{k}+1}^{T}\right) F_{1}\left(e_{n_{1}+n_{3}}-e_{\omega_{\ell}+n_{3}}\right) \\
= & e_{n_{1}+1}^{T} F_{1} e_{n_{1}+n_{3}}-e_{n_{1}+1}^{T} F_{1} e_{\omega_{\ell}+n_{3}}-e_{\omega_{k}+1}^{T} F_{1} e_{n_{1}+n_{3}}+e_{\omega_{k}+1}^{T} F_{1} e_{\omega_{\ell}+n_{3}}=\sum_{j=3}^{\min (k, \ell)} \epsilon_{j} \overline{s_{j, k}} s_{j, \ell}
\end{aligned}
$$

for the same reason. Then, recalling (4.5) and that $f_{n_{3}}$ is Hermitian, we have determined $f_{n_{3}}\left(z_{k}, z_{\ell}\right)$ for all $k$ and $\ell$ in this case. Thus, to extract the signs of (4.4) at blocks of size $n_{3}$, we consider that the matrix $M:=\left[\sum_{j=3}^{\min (k, \ell)} \epsilon_{j} \overline{s_{j, k}} s_{j, \ell}\right]_{k \ell}$ is $*$-congruent (employing the same transformations that were described detail in the treatment of blocks of type (i)) to the diagonal matrix

$$
\operatorname{diag}\left(\epsilon_{3}\left|s_{3,3}\right|^{2}, \epsilon_{4}\left|s_{4,4}\right|^{2}, \ldots, \epsilon_{\tau}\left|s_{\tau, \tau}\right|^{2}\right) .
$$

Hence, since the matrix representation of $f_{n_{3}}$ is given by $M$, the signs of (4.4) at blocks of size $n_{3}$ are clearly given by $\mathcal{L}_{n_{3}}^{\prime \prime}=\left\{\epsilon_{3}, \ldots, \epsilon_{\tau}\right\}$, i.e. the original signs.

Case (b): Distinguishing as before, we start assuming that $k$ and $\ell$ are both odd, then $z_{k}^{*} F_{1} z_{\ell}^{\left(n_{3}\right)}$ is given by:

$$
\begin{aligned}
& \left(e_{1}^{T}-e_{\omega_{k}+1}^{T}-\overline{x_{1}} e_{n_{1}+1}^{T}\right) F_{1}\left(e_{n_{3}}-e_{\omega_{\ell}+n_{3}}-x_{1} e_{n_{1}+n_{3}}-\cdots-x_{n_{3}} e_{n_{1}+1}\right) \\
= & e_{1}^{T} F_{1} e_{n_{3}}-e_{1}^{T} F_{1} e_{\omega_{\ell}+n_{3}}-x_{1} e_{1}^{T} F_{1} e_{n_{1}+n_{3}}-\cdots-x_{n_{3}} e_{1}^{T} F_{1} e_{n_{1}+1} \\
& -e_{\omega_{k}+1}^{T} F_{1} e_{n_{3}}+e_{\omega_{k}+1}^{T} F_{1} e_{\omega_{\ell}+n_{3}}+x_{1} e_{\omega_{k}+1}^{T} F_{1} e_{n_{1}+n_{3}}+\cdots+x_{n_{3}} e_{\omega_{k}+1}^{T} F_{1} e_{n_{1}+1} \\
& -\overline{x_{1}} e_{n_{1}+1}^{T} F_{1} e_{n_{3}}+\overline{x_{1}} e_{n_{1}+1}^{T} F_{1} e_{\omega_{\ell}+n_{3}}+\left|x_{1}\right|^{2} e_{n_{1}+1}^{T} F_{1} e_{n_{1}+n_{3}}+\cdots+\bar{x}_{1} x_{n_{3}} e_{n_{1}+1}^{T} F_{1} e_{n_{1}+1} \\
= & \sum_{j=3}^{\min (k, \ell)} \epsilon_{j} \overline{s_{j, k}} s_{j, \ell}+\epsilon_{2} \overline{\left(s_{2, k}+x_{1} s_{2,2}\right)}\left(s_{2, \ell}+x_{1} s_{2,2}\right) .
\end{aligned}
$$


This result is obtained since

$$
\begin{array}{cc}
e_{\omega_{k}+1}^{T} F_{1} e_{\omega_{\ell}+n_{3}}=\sum_{j=3}^{\min (k, \ell)} \epsilon_{j} \overline{s_{j, k}} s_{j, \ell}+\epsilon_{2} \overline{s_{2, k}} s_{2, \ell}, & x_{1} e_{\omega_{k}+1}^{T} F_{1} e_{n_{1}+n_{3}}=\epsilon_{2} x_{1} \overline{s_{2, k}} s_{2,2}, \\
\bar{x}_{1} e_{n_{1}+1}^{T} F_{1} e_{\omega_{\ell}+n_{3}}=\epsilon_{2} \overline{x_{1} s_{2,2}} s_{2, \ell}, & \left|x_{1}\right|^{2} e_{n_{1}+1}^{T} F_{1} e_{n_{1}+n_{3}}=\epsilon_{2}\left|x_{1} s_{2,2}\right|^{2},
\end{array}
$$

and all other terms in the computation are equal to zero. Then again, if $k$ is odd and $\ell$ is even, $z_{k}^{*} F_{1} z_{\ell}^{\left(n_{3}\right)}$ is equal to

$$
\begin{aligned}
& \left(e_{1}^{T}-e_{\omega_{k}+1}^{T}-\overline{x_{1}} e_{n_{1}+1}^{T}\right) F_{1}\left(e_{n_{1}+n_{3}}-e_{\omega_{\ell}+n_{3}}\right)=e_{1}^{T} F_{1} e_{n_{1}+n_{3}}-e_{1}^{T} F_{1} e_{\omega_{\ell}+n_{3}} \\
& -e_{\omega_{k}+1}^{T} F_{1} e_{n_{1}+n_{3}}+e_{\omega_{k}+1}^{T} F_{1} e_{\omega_{\ell}+n_{3}}-\overline{x_{1}} e_{n_{1}+1}^{T} F_{1} e_{n_{1}+n_{3}}+\overline{x_{1}} e_{n_{1}+1}^{T} F_{1} e_{\omega_{\ell}+n_{3}} \\
= & \sum_{j=3}^{\min (k, \ell)} \epsilon_{j} \overline{s_{j, k}} s_{j, \ell}+\epsilon_{2} \overline{\left(s_{2, k}+x_{1} s_{2,2}\right)}\left(s_{2, \ell}-s_{2,2}\right),
\end{aligned}
$$

since

$$
\begin{aligned}
& -e_{\omega_{k}+1}^{T} F_{1} e_{n_{1}+n_{3}}=-\epsilon_{2} \overline{s_{2, k}} s_{2,2}, \quad e_{\omega_{k}+1}^{T} F_{1} e_{\omega_{\ell}+n_{3}}=\sum_{j=3}^{\min (k, \ell)} \epsilon_{j} \overline{s_{j, k}} s_{j, \ell}+\epsilon_{2} \overline{s_{2, k}} s_{2, \ell}, \\
& -\overline{x_{1}} e_{n_{1}+1}^{T} F_{1} e_{n_{1}+n_{3}}=-\epsilon_{2} \overline{x_{1}}\left|s_{2,2}\right|^{2}, \quad \overline{x_{1}} e_{n_{1}+1}^{T} F_{1} e_{\omega_{\ell}+n_{3}}=\epsilon_{2} \overline{x_{1} s_{2,2}} s_{2, \ell},
\end{aligned}
$$

and all other terms are zero. At last, if $k$ and $\ell$ are both even, then $z_{k}^{*} F_{1} z_{\ell}^{\left(n_{3}\right)}$ is given by

$$
\begin{aligned}
& \left(e_{n_{1}+1}^{T}-e_{\omega_{k}+1}^{T}\right) F_{1}\left(e_{n_{1}+n_{3}}-e_{\omega_{\ell}+n_{3}}\right)=e_{n_{1}+1}^{T} F_{1} e_{n_{1}+n_{3}}-e_{n_{1}+1}^{T} F_{1} e_{\omega_{\ell}+n_{3}}-e_{\omega_{k}+1}^{T} F_{1} e_{n_{1}+n_{3}} \\
& +e_{\omega_{k}+1}^{T} F_{1} e_{\omega_{\ell}+n_{3}}=\epsilon_{2}\left|s_{2,2}\right|^{2}-\epsilon_{2} \overline{s_{2,2}} s_{2, \ell}-\epsilon_{2} \overline{s_{2, k}} s_{2,2}+\sum_{j=3}^{\min (k)} \epsilon_{j} \overline{s_{j, k}} s_{j, \ell}+\epsilon_{2} \overline{s_{2, k}} s_{2, \ell} \\
& =\sum_{j=3}^{\min (k, \ell)} \epsilon_{j} \overline{s_{j, k}} s_{j, \ell}+\epsilon_{2} \overline{\left(s_{2, k}-s_{2,2}\right)}\left(s_{2, \ell}-s_{2,2}\right) .
\end{aligned}
$$

Again, as $f_{n_{3}}$ is Hermitian, this concludes the computation of $f_{n_{3}}\left(z_{k}, z_{\ell}\right)$ for all $k$ and $\ell$ in this case. Now, let us define the matrix $B$ such that the matrix representation of $f_{n_{3}}$ with respect to the above basis is given by $M+\epsilon_{2} B$ (where $M$ is defined as in case (a)). Further, we point out that $B$ has the form $w w^{*}$ for a suitable $w \in \mathbb{C}^{\tau-2}$. Thus, assuming the matrix representation $M+\epsilon_{2} B$ to be invertible (which is a generic condition with respect to the entries of $u, v$ ), we can apply Lemma 2.12. Recalling that the signs of the eigenvalues of $X$ can be read off from (4.9), by this lemma the desired list of signs $\mathcal{L}_{n_{3}}^{\prime \prime}$ attached to blocks of size $n_{3}$ is obtained by removing either exactly one sign -1 or exactly one sign +1 from the list $\mathcal{L}_{n_{3}}=\left\{\epsilon_{2}, \ldots, \epsilon_{\tau}\right\}$.

Case (c): Whenever $k$ and $\ell$ are both odd, $z_{k}^{*} F_{1} z_{\ell}^{\left(n_{3}\right)}$ is given by:

$$
\begin{aligned}
& \left(e_{1}^{T}-e_{\omega_{k}+1}^{T}-\overline{x_{1}} e_{n_{1}+1}^{T}\right) F_{1}\left(e_{n_{3}}-e_{\omega_{\ell}+n_{3}}-x_{1} e_{n_{1}+n_{3}}-\cdots-x_{n_{3}} e_{n_{1}+1}\right) \\
= & e_{1}^{T} F_{1} e_{n_{3}}-e_{1}^{T} F_{1} e_{\omega_{\ell}+n_{3}}-x_{1} e_{1}^{T} F_{1} e_{n_{1}+n_{3}}-\cdots-x_{n_{3}} e_{1}^{T} F_{1} e_{n_{1}+1} \\
& -e_{\omega_{k}+1}^{T} F_{1} e_{n_{3}}+e_{\omega_{k}+1}^{T} F_{1} e_{\omega_{\ell}+n_{3}}+x_{1} e_{\omega_{k}+1}^{T} F_{1} e_{n_{1}+n_{3}}+\cdots+x_{n_{3}} e_{\omega_{k}+1}^{T} F_{1} e_{n_{1}+1} \\
& -\overline{x_{1}} e_{n_{1}+1}^{T} F_{1} e_{n_{3}}+\overline{x_{1}} e_{n_{1}+1}^{T} F_{1} e_{\omega_{\ell}+n_{3}}+\left|x_{1}\right|^{2} e_{n_{1}+1}^{T} F_{1} e_{n_{1}+n_{3}}+\cdots+\overline{x_{1}} x_{n_{3}} e_{n_{1}+1}^{T} F_{1} e_{n_{1}+1} \\
= & \sum_{j=3}^{\min (k, \ell)} \epsilon_{j} \overline{s_{j, k}} s_{j, \ell}+\epsilon_{2} \overline{\left(s_{2, k}+x_{1} s_{2,2}\right)}\left(s_{2, \ell}+x_{1} s_{2,2}\right)+\epsilon_{1} \overline{\left(s_{1, k}-s_{1,1}+x_{1} s_{1,2}\right)}\left(s_{1, \ell}-s_{1,1}+x_{1} s_{1,2}\right) .
\end{aligned}
$$


To obtain this, we have to consider all nonzero terms in the above computation, namely:

$$
\begin{gathered}
e_{1}^{T} F_{1} e_{n_{3}}=\epsilon_{1}\left|s_{1,1}\right|^{2}, \quad-e_{1}^{T} F_{1} e_{\omega_{\ell}+n_{3}}=-\epsilon_{1} \overline{s_{1,1}} s_{1, \ell}, \quad-x_{1} e_{1}^{T} F_{1} e_{n_{1}+n_{3}}=-\epsilon_{1} x_{1} \overline{s_{1,1}} s_{1,2}, \\
-e_{\omega_{k}+1}^{T} F_{1} e_{n_{3}}=-\epsilon_{1} \overline{s_{1, k}} s_{1,1}, \quad e_{\omega_{k}+1}^{T} F_{1} e_{\omega_{\ell}+n_{3}}=\sum_{j=3}^{\min (k, \ell)} \epsilon_{j} \overline{s_{j, k}} s_{j, \ell}+\epsilon_{2} \overline{s_{2, k}} s_{2, \ell}+\epsilon_{1} \overline{s_{1, k}} s_{1, \ell}, \\
x_{1} e_{\omega_{k}+1}^{T} F_{1} e_{n_{1}+n_{3}}=\epsilon_{2} x_{1} \overline{s_{2, k}} s_{2,2}+\epsilon_{1} x_{1} \overline{s_{1, k}} s_{1,2}, \\
\overline{x_{1}} e_{n_{1}+1}^{T} F_{1} e_{\omega_{\ell}+n_{3}}=\epsilon_{2} \overline{x_{1} s_{2,2}} s_{2, \ell}+\epsilon_{1} \overline{x_{1} s_{1,2}} s_{1, \ell}, \\
\left|x_{1}\right|^{2} e_{n_{1}+1}^{T} F_{1} e_{n_{1}+n_{3}}=\epsilon_{2}\left|x_{1} e_{2,2}\right|^{2}+\epsilon_{1}\left|x_{1} s_{1,2}\right|^{2} .
\end{gathered}
$$

Similarly, if $k$ is odd and $\ell$ is even, $z_{k}^{*} F_{1} z_{\ell}^{\left(n_{3}\right)}$ is equal to

$$
\begin{aligned}
& \left(e_{1}^{T}-e_{\omega_{k}+1}^{T}-\overline{x_{1}} e_{n_{1}+1}^{T}\right) F_{1}\left(e_{n_{1}+n_{3}}-e_{\omega_{\ell}+n_{3}}\right)=e_{1}^{T} F_{1} e_{n_{1}+n_{3}}-e_{1}^{T} F_{1} e_{\omega_{\ell}+n_{3}} \\
& -e_{\omega_{k}+1}^{T} F_{1} e_{n_{1}+n_{3}}+e_{\omega_{k}+1}^{T} F_{1} e_{\omega_{\ell}+n_{3}}-\overline{x_{1}} e_{n_{1}+1}^{T} F_{1} e_{n_{1}+n_{3}}+\overline{x_{1}} e_{n_{1}+1}^{T} F_{1} e_{\omega_{\ell}+n_{3}} \\
= & \epsilon_{1} \overline{s_{1,1}} s_{1,2}-\epsilon_{1} \overline{s_{1,1}} s_{1, \ell}-\epsilon_{2} \overline{s_{2, k}} s_{2,2}-\epsilon_{1} \overline{s_{1, k}} s_{1,2}+\sum_{j=1}^{\min (k, \ell)} \epsilon_{j} \overline{s_{j, k}} s_{j, \ell} \\
= & \quad-\epsilon_{2} \overline{x_{1}}\left|s_{2,2}\right|^{2}-\epsilon_{1} \overline{x_{1}}\left|s_{1,2}\right|^{2}+\epsilon_{2} \overline{x_{1} s_{2,2}} s_{2, \ell}+\epsilon_{1} \overline{x_{1} s_{1,2}} s_{1, \ell} \\
& \sum_{j=3}^{\min (k, \ell)} \epsilon_{j} \overline{s_{j, k}} s_{j, \ell}+\epsilon_{2} \overline{\left(s_{2, k}+x_{1} s_{2,2}\right)}\left(s_{2, \ell}-s_{2,2}\right)+\epsilon_{1} \overline{\left(s_{1, k}-s_{1,1}+x_{1} s_{1,2}\right)}\left(s_{1, \ell}-s_{1,2}\right) .
\end{aligned}
$$

Finally, if $k$ and $\ell$ are both even, then $z_{k}^{*} F_{1} z_{\ell}^{\left(n_{3}\right)}$ is given by

$$
\begin{aligned}
& \left(e_{n_{1}+1}^{T}-e_{\omega_{k}+1}^{T}\right) F_{1}\left(e_{n_{1}+n_{3}}-e_{\omega_{\ell}+n_{3}}\right) \\
= & e_{n_{1}+1}^{T} F_{1} e_{n_{1}+n_{3}}-e_{n_{1}+1}^{T} F_{1} e_{\omega_{\ell}+n_{3}}-e_{\omega_{k}+1}^{T} F_{1} e_{n_{1}+n_{3}}+e_{\omega_{k}+1}^{T} F_{1} e_{\omega_{\ell}+n_{3}} \\
= & \epsilon_{2}\left|s_{2,2}\right|^{2}+\epsilon_{1}\left|s_{1,2}\right|^{2}-\epsilon_{2} \overline{s_{2,2}} s_{2, \ell}-\epsilon_{1} \overline{s_{1,2}} s_{1, \ell}-\epsilon_{2} \overline{s_{2, k}} s_{2,2}-\epsilon_{1} \overline{s_{1, k}} s_{1,2}+\sum_{j=1}^{\min (k, \ell)} \epsilon_{j} \overline{s_{j, k}} s_{j, \ell} \\
= & \sum_{j=3}^{\min (k, \ell)} \epsilon_{j} \overline{s_{j, k}} s_{j, \ell}+\epsilon_{2} \overline{\left(s_{2, k}-s_{2,2}\right)}\left(s_{2, \ell}-s_{2,2}\right)+\epsilon_{1} \overline{\left(s_{1, k}-s_{1,2}\right)}\left(s_{1, \ell}-s_{1,2}\right) .
\end{aligned}
$$

As in the previous cases, this concludes the computation of $f_{n_{3}}\left(z_{k}, z_{\ell}\right)$ for all $k$ and $\ell$. Now, we define the matrix $C$ such that the matrix representation of $f_{n_{3}}$ with respect to the above basis is given by $M+\epsilon_{2} B+\epsilon_{1} C$ in this case (where $M$ and $B$ are defined as before). Then, also $C$ has the form $w w^{*}$ for some $w \in \mathbb{C}^{\tau-2}$. Clearly, assuming the generic condition that both $M+\epsilon_{2} B$ and $M+\epsilon_{2} B+\epsilon_{1} C$ are invertible, we may apply Lemma 2.12 twice, so that the desired list of signs $\mathcal{L}_{n_{3}}^{\prime \prime}$ is obtained by removing either exactly two signs $-1,-1$ or exactly two signs $-1,+1$ or exactly two signs $+1,+1$ from the list $\mathcal{L}_{n_{3}}=\left\{\epsilon_{1}, \ldots, \epsilon_{\tau}\right\}$.

\section{Conclusion}

The canonical form of regular Hermitian matrix pencils was investigated under generic structure-preserving rank- 1 and rank- 2 perturbations. Hereby, regarding the sizes of the Jordan blocks, a generic Hermitian rank-1 or rank-2 perturbation does not differ from 
a generic unstructured rank-1 or rank-2 perturbation: At each eigenvalue $\hat{\lambda}$, the largest one or two, respectively, Jordan blocks are destroyed and in addition, if $\hat{\lambda}$ is a (simple) eigenvalue of the perturbation, a new block of size one is created. In addition, if $\hat{\lambda}$ is real (or infinite), under a rank-1 or rank-2 perturbation, all but one or two, respectively, of the signs at each eigenvalue are preserved, whereby the signs that are not preserved correspond to blocks that have been destroyed.

Finally, the sign of the potential new block of size one at $\widehat{\lambda}$ can be determined as follows (in case it is real or infinite): If there exist blocks of size greater than one in the unperturbed pencil at $\widehat{\lambda}$, then generically the sign that is attached to $\widehat{\lambda}$ in the perturbation is added to the list of signs at blocks of size one. On the other hand, if the largest blocks in the unperturbed pencil at $\hat{\lambda}$ have size one, then the list of signs at these blocks is generically changed as follows: First, the sign that is attached to $\widehat{\lambda}$ in the perturbation is added, and then exactly one sign -1 or +1 is removed from that list.

\section{Acknowledgement}

The author is grateful to Christian Mehl for his careful reading of this manuscript.

\section{References}

[1] R. Alam, S. Bora, M. Karow, V. Mehrmann, and J. Moro. Perturbation theory for Hamiltonian matrices and the distance to bounded-realness. SIAM J. Matrix Anal. Appl., 432:484-514, 2011.

[2] L. Batzke. Generic rank-two perturbations of structured regular matrix pencils. Preprint series of the Institute of Mathematics, Technische Universität Berlin, Preprint 09-2014. Submitted for publication, 2014.

[3] L. Batzke. Generic rank-one perturbations of structured regular matrix pencils. Linear Algebra Appl., 458:638-670, 2014.

[4] F. De Terán, F. Dopico, and J. Moro. Low rank perturbation of weierstrass structure. SIAM J. Matrix Anal. Appl., 30:538-547, 2008.

[5] J.H. Fourie, G.J. Groenewald, D.B. Janse van Rensburg, and A.C.M. Ran. Rank one perturbations of H-positive real matrices. Linear Algebra Appl., 439(3):653-674, 2013.

[6] F.R. Gantmacher. Theory of Matrices, volume 2. Chelsea, New York, 1959.

[7] F.R. Gantmacher. Theory of Matrices, volume 1. Chelsea, New York, 1959.

[8] I. Gohberg, P. Lancaster, and L. Rodman. Matrices and Indefinite Scalar Products. Birkhäuser, Basel, 1983. 
[9] I. Gohberg, P. Lancaster, and L. Rodman. Indefinite Linear Algebra and Applications. Birkhäuser, Basel, 2005.

[10] L. Hörmander and A. Melin. A remark on perturbations of compact operators. Math. Scand., 167:113-118, 1994.

[11] R.A. Horn and C.R. Johnson. Matrix Analysis. Cambridge University Press, Cambridge, 1985.

[12] P. Lancaster and L. Rodman. Canonical forms for hermitian matrix pairs under strict equivalence and congruence. SIAM Review, 47(3):407-443, 2005.

[13] P. Lancaster and M. Tismenetsky. The Theory of Matrices. Academic Press, Orlando, 2nd edition, 1985.

[14] C. Mehl, V. Mehrmann, A.C.M. Ran, and L. Rodman. Eigenvalue perturbation theory of classes of structured matrices under generic structured rank one perturbations. Linear Algebra Appl., 435:687-716, 2011.

[15] C. Mehl, V. Mehrmann, A.C.M. Ran, and L. Rodman. Perturbation theory of selfadjoint matrices and sign characteristics under generic structured rank one perturbations. Linear Algebra Appl., 436:4027-4042, 2012.

[16] C. Mehl, V. Mehrmann, A.C.M. Ran, and L. Rodman. Jordan forms of real and complex matrices under rank one perturbations. Oper. Matrices, 7:381-398, 2013.

[17] C. Mehl, V. Mehrmann, A.C.M. Ran, and L. Rodman. Eigenvalue perturbation theory of symplectic, orthogonal, and unitary matrices under generic structured rank one perturbations. BIT, 54:219-255, 2014.

[18] V. Mehrmann and H. Xu. Perturbation of purely imaginary eigenvalues of hamiltonian matrices under structured perturbations. Electron. J. Linear Algebra, 17:234-257, 2008.

[19] J. Moro and F. Dopico. Low rank perturbation of jordan structure. SIAM J. Matrix Anal. Appl., 25:495-506, 2003.

[20] S.V. Savchenko. Typical changes in spectral properties under perturbations by a rankone operator. Mat. Zametki, 74:590-602, 2003. (Russian). Translation in Mathematical Notes. 74:557-568, 2003.

[21] S.V. Savchenko. On the change in the spectral properties of a matrix under a perturbation of sufficiently low rank. Funkts. Anal. Prilozh., 38:85-88, 2004. (Russian). Translation in Funct. Anal. Appl. 38:69-71, 2004.

[22] S.V. Savchenko. Laurent expansion for the determinant of the matrix of scalar resolvents. Mathematicheskii Sbornik, 196:121-144, 2005. (Russian). Translation in Sbornik: Mathematics. 196:743-764, 2005. 


\section{Appendix}

The following matrix is denoted by $T$ and we want to compute its determinant:

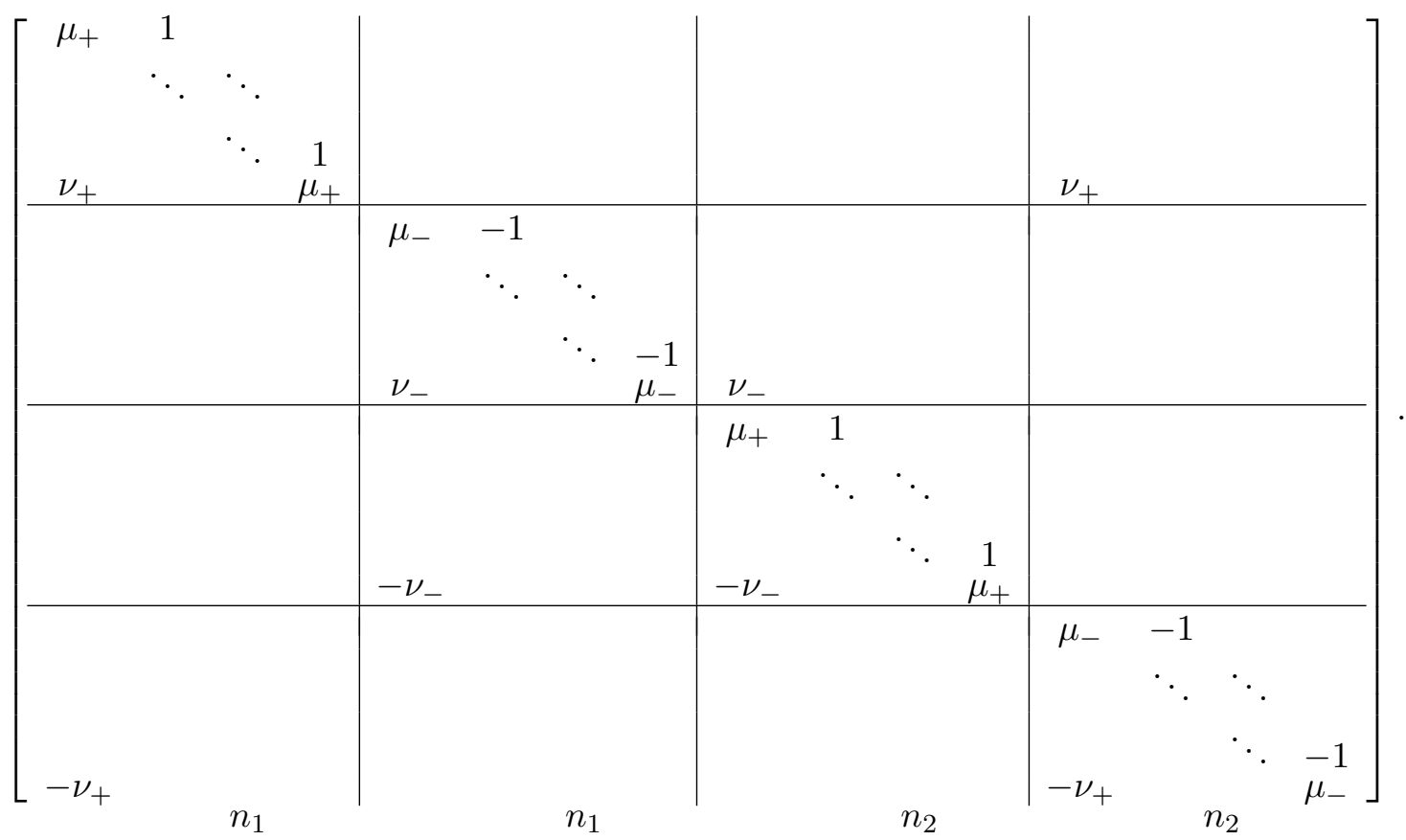

Laplace expansion with respect to the first column gives

$$
\operatorname{det} T=\mu_{+} \operatorname{det} T_{1}+(-1)^{n_{1}+1} \nu_{+} \operatorname{det} T_{2}+\nu_{+} \operatorname{det} T_{3},
$$

where

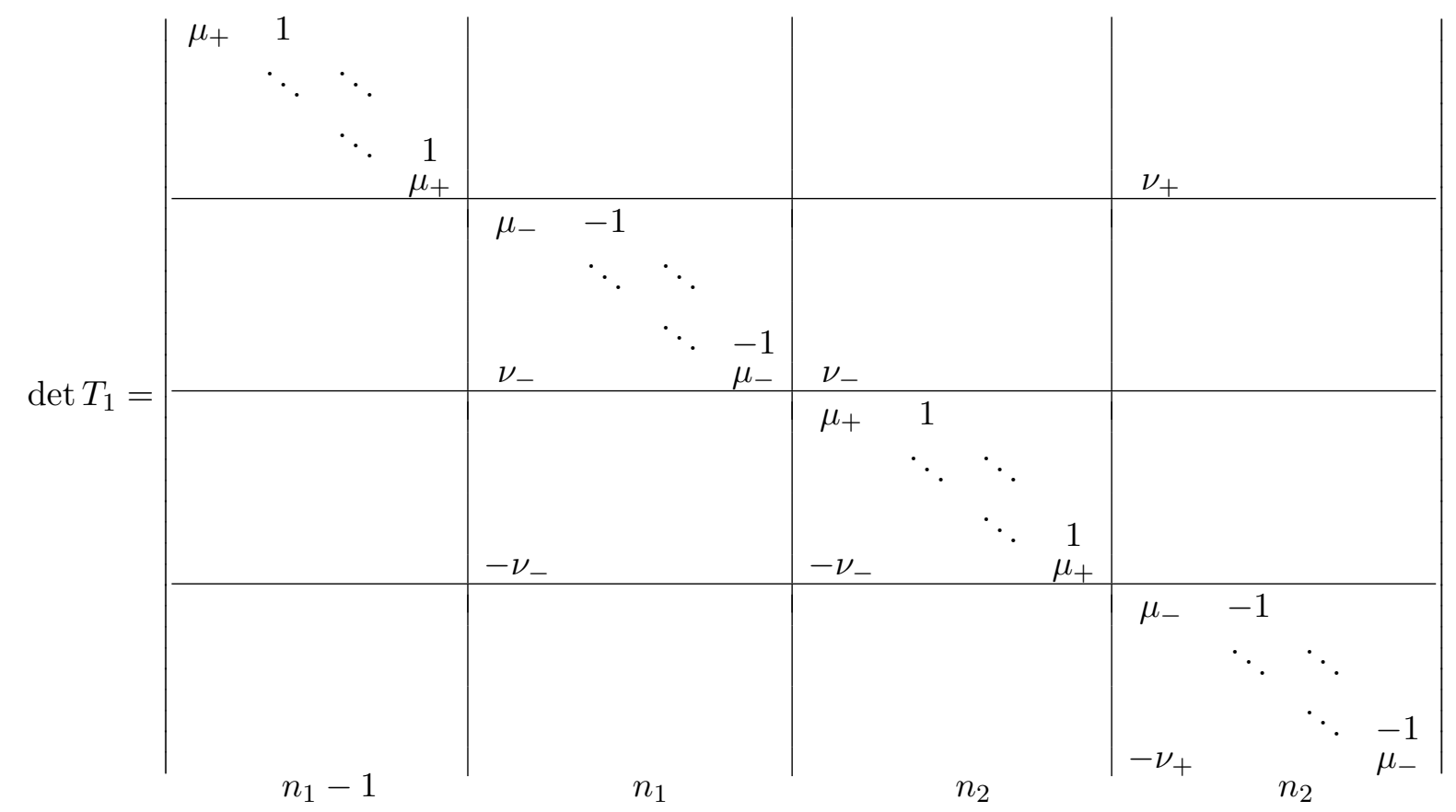




$$
=\mu_{+}^{n_{1}-1}\left(\mu_{-}^{n_{2}}-\nu_{+}\right) \operatorname{det} T_{m i d},
$$

denoting by $T_{\text {mid }}$ the middle $\left(n_{1}+n_{2}\right) \times\left(n_{1}+n_{2}\right)$ block of $T$. Moreover, we have

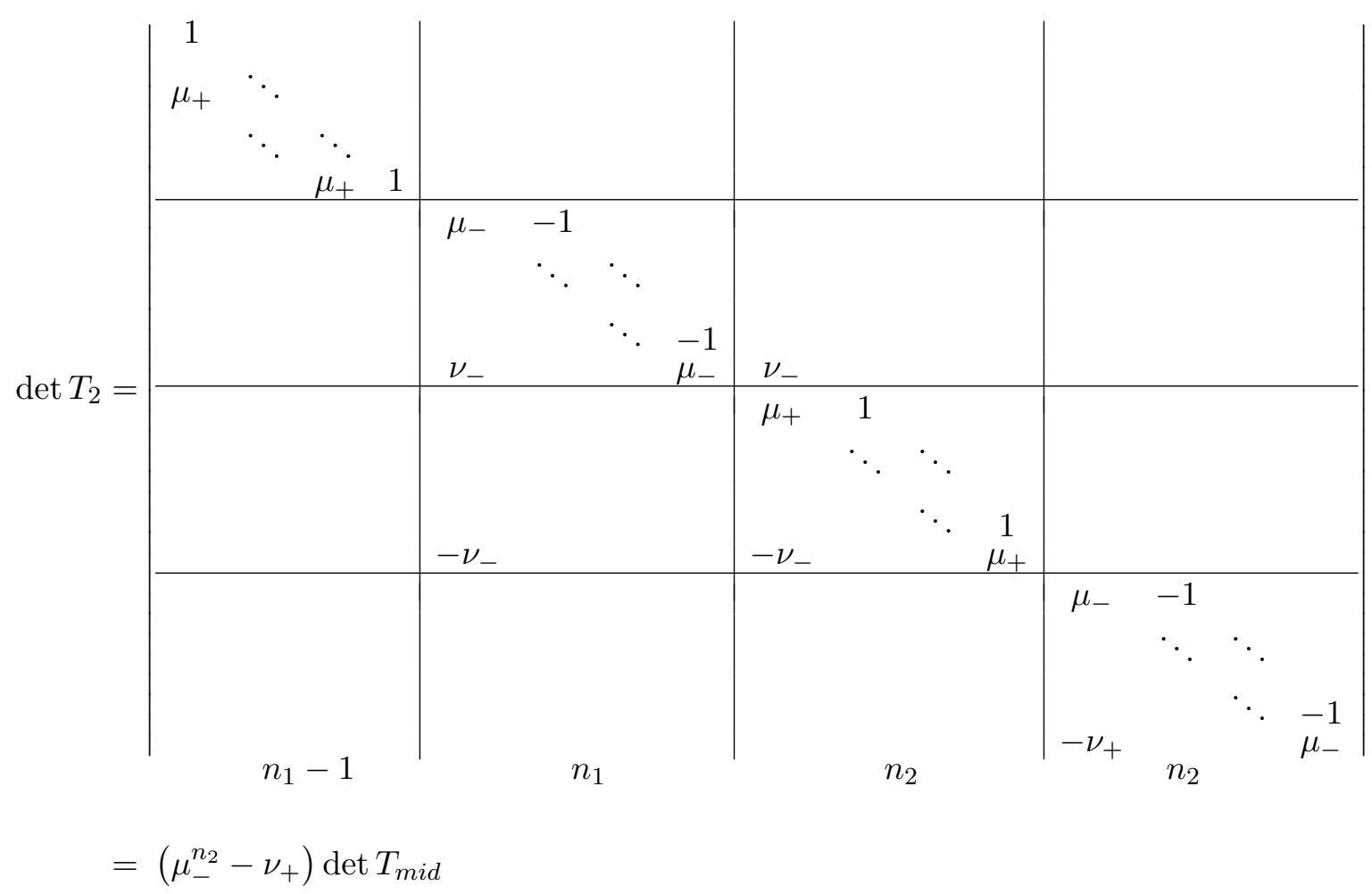

and

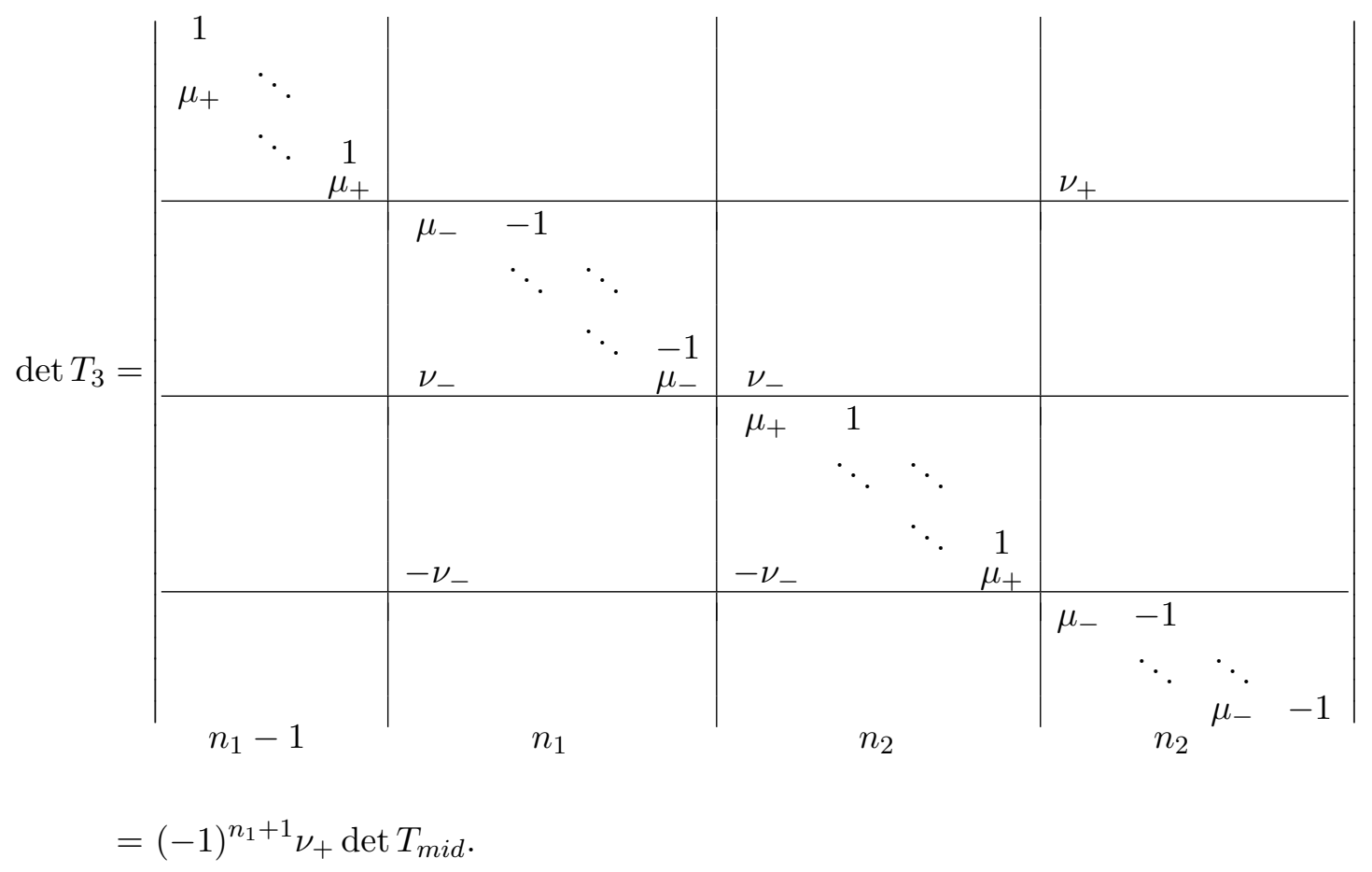


Putting these computations together, we obtain

$$
\begin{aligned}
\operatorname{det} T & =\left[\mu_{+}^{n_{1}}\left(\mu_{-}^{n_{2}}-\nu_{+}\right)+(-1)^{n_{1}+1} \nu_{+}\left(\mu_{-}^{n_{2}}-\nu_{+}\right)+\nu_{+}^{2}(-1)^{n_{1}+1}\right] \operatorname{det} T_{\text {mid }} \\
& =\left[\mu_{+}^{n_{1}} \mu_{-}^{n_{2}}-\mu_{+}^{n_{1}} \nu_{+}-(-1)^{n_{1}} \mu_{-}^{n_{2}} \nu_{+}\right] \operatorname{det} T_{\text {mid }} .
\end{aligned}
$$

We continue with computing

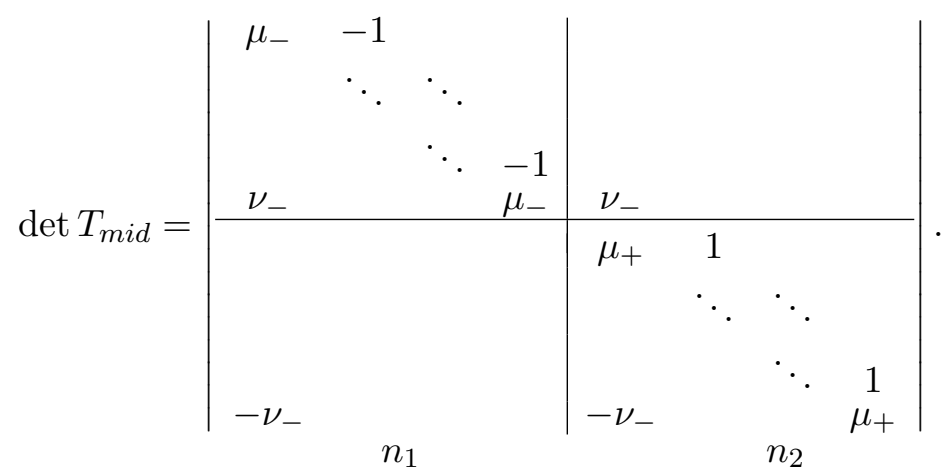

A Laplace expansion with respect to the first column yields

$$
\operatorname{det} T_{\text {mid }}=\mu_{-} \operatorname{det} T_{4}+(-1)^{n_{1}+1} \nu_{-} \operatorname{det} T_{5}+(-1)^{n_{1}+n_{2}} \nu_{-} \operatorname{det} T_{6},
$$

where

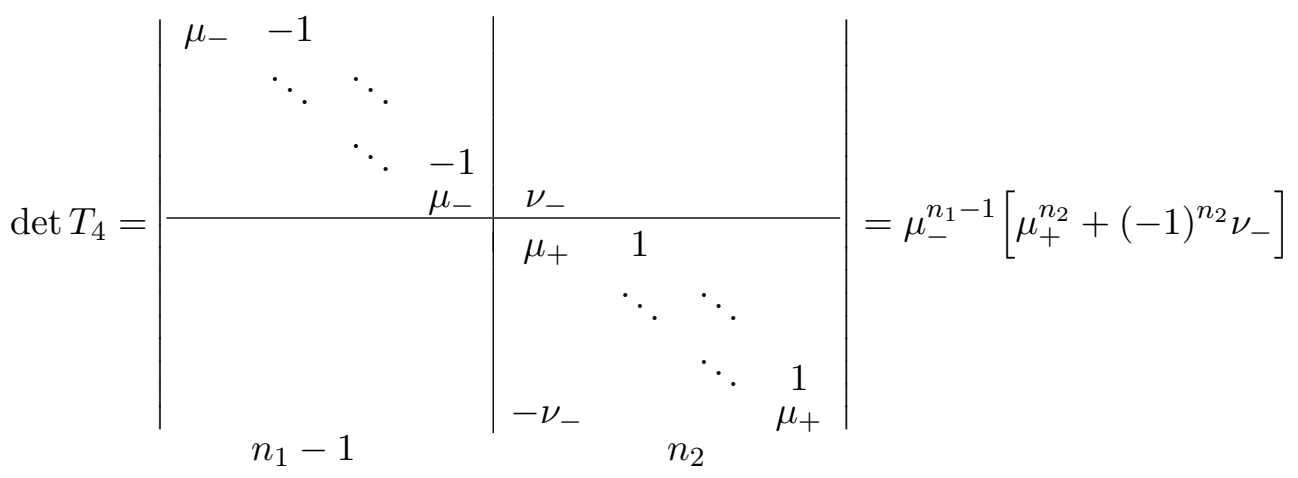

and

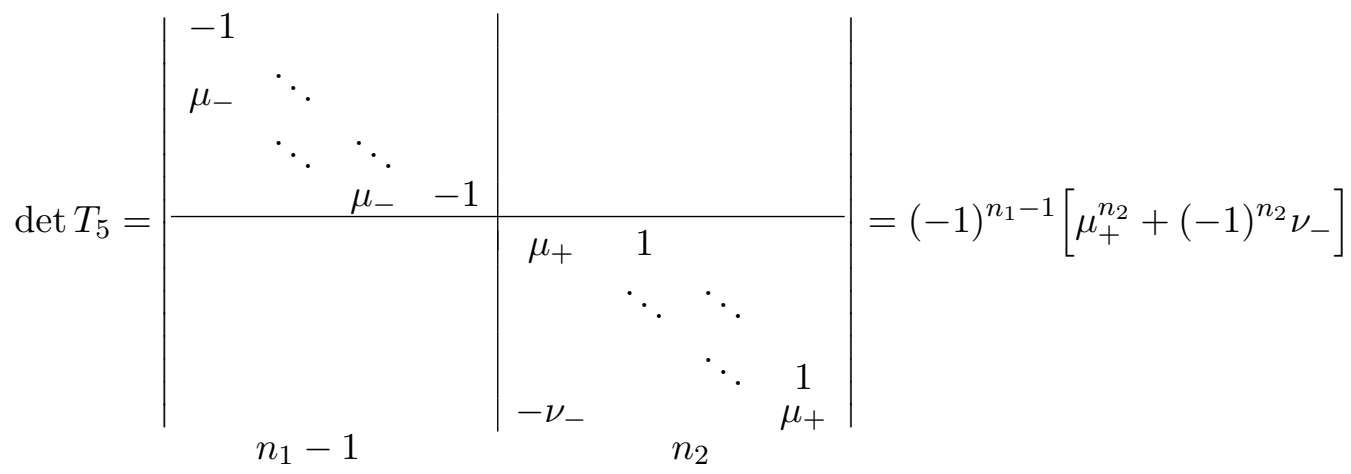


as well as

$$
\operatorname{det} T_{6}=\left|\begin{array}{ccc|ccc}
-1 & & & & & \\
\mu_{-} & \ddots & & & & \\
& \ddots & -1 & & & \\
& & \mu_{-} & \nu_{-} & & \\
\hline & & & \mu_{+} & 1 & \\
& & \ddots & \ddots & \\
& & & \mu_{+} & 1
\end{array}\right|=(-1)^{n_{1}-1} \nu_{-} .
$$

Hence, we obtain

$$
\begin{aligned}
\operatorname{det} T_{\text {mid }} & =\mu_{-}^{n_{1}}\left(\mu_{+}^{n_{2}}+(-1)^{n_{2}} \nu_{-}\right)+\nu_{-}\left(\mu_{+}^{n_{2}}+(-1)^{n_{2}} \nu_{-}\right)+(-1)^{n_{2}+1} \nu_{-}^{2} \\
& =\mu_{-}^{n_{1}} \mu_{+}^{n_{2}}+(-1)^{n_{2}} \mu_{-}^{n_{1}} \nu_{-}+\mu_{+}^{n_{2}} \nu_{-},
\end{aligned}
$$

which altogether yields

$$
\operatorname{det} T=\left[\mu_{+}^{n_{1}} \mu_{-}^{n_{2}}-\mu_{+}^{n_{1}} \nu_{+}-(-1)^{n_{1}} \mu_{-}^{n_{2}} \nu_{+}\right]\left[\mu_{-}^{n_{1}} \mu_{+}^{n_{2}}+(-1)^{n_{2}} \mu_{-}^{n_{1}} \nu_{-}+\mu_{+}^{n_{2}} \nu_{-}\right] .
$$

